\title{
Special families of curves, of Abelian varieties and of certain minimal manifolds over curves
}

\author{
Martin Möller ${ }^{1}$, Eckart Viehweg ${ }^{1}$ and Kang Zuo ${ }^{2 \star}$ \\ 1 Universität Duisburg-Essen, Mathematik, 45117 Essen, Germany \\ martin.moeller@uni-essen.de \\ viehweg@uni-essen.de \\ 2 Universität Mainz, Fachbereich 17, Mathematik, 55099 Mainz, Germany \\ kzuo@mathematik.uni-mainz.de
}

\section{Introduction}

Let $f: X \rightarrow Y$ be a surjective morphism from an $n+1$ dimensional complex projective manifold $X$ to a curve $Y$. In this article we want to present some recent results about the structure of $f$, in particular about its discriminant locus $S$, i.e. the set of points $s$ in $Y$ with $f^{-1}(s)$ singular. So

$$
f: V=f^{-1}(U) \longrightarrow U=Y \backslash S
$$

will always be smooth. We will usually require $\Delta=f^{*} S$ to be a normal crossing divisor.

If $Y=\mathbb{P}^{1}$ and if $X$ is a curve of genus $g(X)>0$ the Hurwitz formula implies that $\# S \geq 3$. In the extremal case $\# S=3, X$ is obviously defined over a number field. G.V. Belyı has shown in [Be79] that the existence of $f$ with $\# S=3$ characterizes curves defined over a number field.

As conjectured by F. Catanese and M. Schneider, the first part can be generalized for $n>0$.

Theorem 0.1 ([VZ01]). Let $X$ be a complex projective manifold of Kodaira dimension $\kappa(X) \geq 0$ and let $f: X \rightarrow \mathbb{P}^{1}$ be a surjective morphism. Then $f$ has at least 3 singular fibres.

It is more reasonable to assume that the general fibre $F$ of $f$ is connected, or as we will say, that $f: X \rightarrow Y$ is a family of $n$-dimensional manifolds, and to use the Hurwitz formula for the Stein factorization to get hold of the general case. $Y$ will be again a curve of arbitrary genus. Putting together results due to Parshin-Arakelov, Migliorini, Kovács, Bedulev-Viehweg, Oguiso-Viehweg and Viehweg-Zuo (see [VZ01] and the references given there) one has:

${ }^{\star}$ This work has been supported by the "DFG-Schwerpunktprogramm Globale Methoden in der Komplexen Geometrie", and by the DFG-Leibniz program. 
Theorem 0.2. Let $f: X \rightarrow Y$ be a non-isotrivial family of $n$-folds, with general fibre $F$. Assume either

a. $\kappa(F)=\operatorname{dim}(F)$, or

b. $\omega_{F}$ semiample.

Then $\operatorname{deg}\left(\Omega_{Y}^{1}(\log S)\right)=2 g(Y)-2+\# S>0$.

Here $f$ is isotrivial, if over some finite covering $Y^{\prime} \rightarrow Y$ the pullback $X \times_{Y} Y^{\prime}$ is birational to $F \times Y^{\prime}$.

As a byproduct, the proof of 0.2 in [VZ01] (see also [Kov02]) gives some explicit lower bounds for the degree of $\operatorname{deg}\left(\Omega_{Y}^{1}(\log S)\right)$. Writing $\delta$ for the number of singular fibres of $f$, which are not semistable, i.e. not reduced normal crossing divisors, one finds constants $\nu$ and $e$, depending only on the Hilbert polynomial $h$ of some polarization of the family, with

$$
\frac{\operatorname{deg}\left(f_{*} \omega_{X / Y}^{\nu}\right)}{\operatorname{rank}\left(f_{*} \omega_{X / Y}^{\nu}\right)} \leq(n \cdot(2 g(Y)-2+\# S)+\delta) \cdot \nu \cdot e .
$$

In Section 2 we will use [VZ05, 3.4] to get a similar bound for arbitrary semistable families.

Theorem 0.3. Assume that $f: X \rightarrow Y$ is a semistable family of $n$-folds. If $Y=\mathbb{P}^{1}$ assume in addition that $\# S \geq 2$. Then for all $\nu \geq 1$ with $f_{*} \omega_{X / Y}^{\nu} \neq 0$

$$
\frac{\operatorname{deg}\left(f_{*} \omega_{X / Y}^{\nu}\right)}{\operatorname{rank}\left(f_{*} \omega_{X / Y}^{\nu}\right)} \leq \frac{n \cdot \nu}{2} \cdot(2 g(Y)-2+\# S)=\frac{n \cdot \nu}{2} \cdot \operatorname{deg}\left(\Omega_{Y}^{1}(\log S)\right) .
$$

The assumption $\# S \geq 0$ is just added to guaranty that $\operatorname{deg}\left(\Omega_{Y}^{1}(\log S)\right)$ is non-negative, and that the fundamental group of $Y \backslash S$ sufficiently large. Of course it will always hold true if one declares one or two smooth fibres to be singular.

There are families of Abelian varieties and of Calabi-Yau manifolds, with

$$
\operatorname{deg}\left(f_{*} \omega_{X / Y}^{\nu}\right)=\frac{\operatorname{deg}\left(f_{*} \omega_{X / Y}^{\nu}\right)}{\operatorname{rank}\left(f_{*} \omega_{X / Y}^{\nu}\right)}=\frac{n \cdot \nu}{2} \cdot \operatorname{deg}\left(\Omega_{Y}^{1}(\log S)\right) .
$$

We do not know, whether there are families of manifolds of higher Kodaira dimension with such an equality.

Theorem 0.3 implies Theorem 0.2. In fact, if $\operatorname{deg}\left(\Omega_{Y}^{1}(\log S)\right) \leq 0$ the open set $U$ is either an elliptic curve or it contains $\mathbb{C}^{*}$. In the first case the family is smooth, in the second one one finds a new family over an étale covering $\mathbb{C}^{*} \rightarrow \mathbb{C}^{*}$, which is semistable. For a semistable family $f: X \rightarrow Y$ Theorem 0.3 implies that $\operatorname{deg}\left(f_{*} \omega_{X / Y}^{\nu}\right) \leq 0$. On the other hand, the assumptions a) or b) in Theorem 0.2 imply that for $\nu \geq 2$

$$
\operatorname{deg}\left(f_{*} \omega_{X / Y}^{\nu}\right) \geq 0
$$


and that $\operatorname{deg}\left(f_{*} \omega_{X / Y}^{\nu}\right)=0$ if and only if $f$ is isotrivial. The same argument is used to show Theorem 0.1:

If there is a morphism with 2 singular fibres, one uses the Hurwitz formula to show that the Stein factorization of $X \rightarrow \mathbb{P}^{1}$ is $\mathbb{P}^{1}$, and that the induced morphism has again at most 2 singular fibres. Here the positivity of $\operatorname{deg}\left(f_{*} \omega_{X / Y}^{\nu}\right)$ follows from $\kappa(X) \geq 0$, and as above one obtains a contradiction to the inequality stated in Theorem 0.3 .

In addition, the bound in Theorem 0.3 implies some weak analogue of the Shafarevich conjecture on the finiteness of smooth families of curves over a fixed curve $U$.

Corollary 0.4. Fix $(Y, S)$ and a polynomial $h$. Then up to deformation there are only finitely many non-isotrivial families $f: X \rightarrow Y$ smooth over $U=$ $Y \backslash S$, such that:

1. $\omega_{F}$ is ample with Hilbert polynomial $h$.

2. $\omega_{F}^{\rho}=\mathcal{O}_{F}$ for some $\rho>0$, and the smooth part $V \rightarrow U$ allows a polarization with Hilbert polynomial $h$.

In fact, consider the moduli scheme $M_{h}$ parameterizing either canonically polarized manifolds, or polarized manifolds with a torsion canonical divisor, and with Hilbert polynomial $h$. In both cases one can show (see [Vi05]) that for a given $\nu \geq 2$ with $h(\nu) \neq 0$ there is a projective compactification $\bar{M}_{h}$ and for some $p$ an invertible sheaf $\lambda_{\nu}^{(p)}$ on $\bar{M}_{h}$ with:

1. $\lambda_{\nu}^{(p)}$ is numerically effective, and ample with respect to $M_{h}$.

2. If $f: X \rightarrow Y$ is a semistable family over a curve $Y$, whose general fibre belongs to the moduli functor, and if $\varphi: Y \rightarrow \bar{M}_{h}$ is the extension of the induced morphism to $M_{h}$, then

$$
\varphi^{*} \lambda_{\nu}^{(p)}=\operatorname{det}\left(f_{*} \omega_{X / Y}^{\nu}\right)^{p} .
$$

Here an invertible sheaf $\lambda$ is "ample with respect to $M_{h}$ " if there exists some modification $\tau: \bar{M}_{\underline{h}}^{\prime} \rightarrow \bar{M}_{h}$, some $\mu \gg 1$ and an effective divisor $E$ on $\bar{M}_{h}^{\prime}$, supported in $\tau^{-1}\left(\bar{M}_{h} \backslash M_{h}\right)$, such that $\tau^{*} \lambda \otimes \mathcal{O}_{\bar{M}_{h}^{\prime}}(-E)$ is ample.

So Theorem 0.3 says that for given $Y$ and $S$ the degree of $\varphi^{*} \lambda_{\nu}^{(p)}$ is bounded, which implies Corollary 0.4. Using a different argument (see [VZ02, 6.2]) one can extend 0.4 to families of minimal models of arbitrary (nonnegative) Kodaira dimension.

As we will see in Section 2 the Theorems 0.1, 0.2 and 0.3 follow from the study of variations of Hodge structures of certain families obtained as cyclic coverings of $X \rightarrow Y$.

It remains the question, what is special about families with few singular fibres. Here the only results are for families controlled by their variation of Hodge structures $R^{n} f_{*} \mathbb{C}_{V}$ (or $R^{1} f_{*} \mathbb{C}_{V}$ for families of Abelian varieties). Moreover, we will assume that the family has semistable reduction, or slightly 
weaker, that the local monodromies in all $s \in S$ are unipotent. Before stating the results, and before giving "few singular fibres" a precise meaning, we will need some notations.

Let $\mathbb{V} \subset R^{k} \mathbb{C}_{V}$ be a $\mathbb{C}$ sub variation of Hodge structures. $\mathbb{V} \otimes_{C} \mathcal{O}_{U}$ extends to a locally free sheaf $\mathcal{H}$ on $Y$ in such a way that the Gauß-Manin connection acquires logarithmic singularities. We choose for $\mathcal{H}$ the Deligne extension, i.e. an extension such that the real part of the local residues are zero. The Hodge filtration extends to a holomorphic filtration on $\mathcal{H}$, and the extended GaußManin connection defines on the associated graded bundle the structure of a logarithmic Higgs bundle $(F, \tau)$, i.e. a locally free sheaf $F$ together with a collection of maps

$$
\tau_{p, q}: F^{p, q} \longrightarrow F^{p-1, q+1} \otimes \Omega_{Y}^{1}(\log S), \quad p+q=k .
$$

The bundle maps $\tau_{p, q}$ can be iterated to obtain

$$
\tau^{(\ell)}: F^{k, 0} \longrightarrow F^{k-1,1} \otimes \Omega_{Y}^{1}(\log S) \longrightarrow \cdots \longrightarrow F^{k-\ell, \ell} \otimes S^{\ell}\left(\Omega_{Y}^{1}(\log S)\right) .
$$

\section{Definition 0.5.}

i. We call $\tau^{(k)}: F^{k, 0} \rightarrow F^{0, k} \otimes S^{k}\left(\Omega_{Y}^{1}(\log S)\right)$ the Griffiths-Yukawa coupling of $\mathbb{V}$ (or of $f$ in case $\mathbb{V}=R^{k} f_{*} \mathbb{C}_{V}$ ).

ii. The Higgs field is strictly maximal, if $F^{k, 0} \neq 0$ and if the $\tau_{p, q}$ are all isomorphisms.

As it will turn out, the property ii) is of numerical nature.

Lemma 0.6 ([VZ05]). Assume that $\mathbb{V}$ is a variation of polarized complex Hodge structures of weight $n$ with unipotent local monodromy in all $s \in S$, and with logarithmic Higgs bundle $\left(\bigoplus F^{p, q}, \tau_{p, q}\right)$. If $F^{k, 0} \neq 0$ one has the Arakelov inequality

$$
\operatorname{deg}\left(F^{k, 0}\right) \leq \frac{k}{2} \cdot \operatorname{rank}\left(F^{k, 0}\right) \cdot \operatorname{deg}\left(\Omega_{Y}^{1}(\log S)\right),
$$

and (1) is an equality if and only if one has a decomposition $\mathbb{V}=\mathbb{V}_{1} \oplus \mathbb{V}_{2}$ where the Higgs field of $\mathbb{V}_{1}$ is strictly maximal and where $\mathbb{V}_{2}$ is a variation of polarized complex Hodge structures, zero in bidegree $(k, 0)$.

For families of curves, the inequality (1) is due to Arakelov, and for families of Abelian varieties it has been shown by Faltings in [Fa83].

If $\# S$ is even, [VZ03, 3.4] gives a more precise description of $\mathbb{V}_{1}$. Choose a logarithmic theta characteristic, i.e. an invertible sheaf $\mathcal{L}$ with $\mathcal{L}^{2}=$ $\Omega_{Y}^{1}(\log S)$. The existence of $\mathbb{V}$ in 0.6 implies that $\operatorname{deg}\left(\Omega_{Y}^{1}(\log S)>0\right.$. Then the Higgs bundle $\mathcal{L} \oplus \mathcal{L}^{-1}$ with Higgs field

$$
\mathcal{L} \underset{\simeq}{\stackrel{\tau}{\longrightarrow}} \mathcal{L}^{-1} \otimes \Omega_{Y}^{1}(\log S)
$$

is stable. As recalled in Section 1 it must be the Higgs bundle of a polarized variation of Hodge structures $\mathbb{L}$ of weight one. $\mathbb{L}$ is unique up to the tensor 
product with a unitary rank one local system, induced by a two-division point of $\operatorname{Pic}^{0}(Y)$.

Lemma 0.7. Assume that $\# S$ is even, and that $\mathbb{V}_{1}$ is a variation of Hodge structures with a strictly maximal Higgs field and with unipotent local monodromies. Then there exists a unitary local system $\mathbb{T}$ on $Y$, regarded as a variation of Hodge structures in bidegree $(0,0)$, with $\mathbb{V}_{1}=\left.S^{k}(\mathbb{L}) \otimes_{C} \mathbb{T}\right|_{U}$.

So "special families" or "families with few singular fibres" will be those where the variation of Hodge structures $R^{k} \mathbb{C}_{V}$ contains an irreducible sub variation $\mathbb{V}$ with a strictly maximal Higgs field, or equivalently a sub variation $\mathbb{V}$ for whose Higgs bundle the equality

$$
\operatorname{deg}\left(F^{k, 0}\right)=\frac{k}{2} \cdot \operatorname{rank}\left(F^{k, 0}\right) \cdot \operatorname{deg}\left(\Omega_{Y}^{1}(\log S)\right)
$$

holds. Here $k=n$, except for families of Abelian varieties, where we usually choose $k=1$.

Let us first consider a semistable family $f: X \rightarrow Y$ of Abelian varieties, smooth over $U=Y \backslash S$, and $V=f^{-1}(U)$.

Theorem 0.8 ([VZ04]). Assume that each irreducible and non-unitary sub variation $\mathbb{V}$ of Hodge structures in $R^{1} f_{*} \mathbb{C}_{V}$ has a strictly maximal Higgs field. Then (replacing $U$ by an étale covering and $V$ by the pullback family) $U$ is a Shimura curve of Hodge type, and $f: V \rightarrow U$ the corresponding universal family.

The construction of Shimura curves will be recalled in Section 3. Let us just mention here, that a Shimura curve $U$ is an étale covering of a certain moduli space of Abelian varieties with prescribed Mumford-Tate group and a suitable level structure. So whenever we talk about Shimura curves, this is a property up to étale coverings of $U$. This allows in particular to assume that \#S is even.

As we will recall in Section 4 the converse of Theorem 0.8 was shown in [Moe3]:

Theorem 0.9. If $f: V \rightarrow U$ is the universal family over a Shimura curve its Higgs field is strictly maximal.

Because of Kollár's decomposition [Kol87] (see Lemma 1.9 below) one can write $f_{*} \Omega_{X / Y}^{1}(\log S)$ as a direct sum of an ample sheaf $\mathcal{A}$ and a subsheaf $\mathcal{B}$, flat for the Gauß-Manin connection. Correspondingly on the local system side one has a decomposition $R^{1} f_{*} \mathbb{C}_{V}=\mathbb{W} \oplus \mathbb{U}$, where $\mathbb{U}$ is unitary and invariant under complex conjugation, and where the $(1,0)$ component of $\mathbb{W}$ is ample. Hence the Higgs bundle corresponding to $\mathbb{W}$ is of the form $\left(\mathcal{A} \oplus \mathcal{A}^{\vee}, \theta\right)$ and using 0.6 one can restate the Theorem 0.8 as:

If $\operatorname{deg}(\mathcal{A})=\frac{1}{2} \cdot \operatorname{rank}(\mathcal{A}) \cdot \operatorname{deg}\left(\Omega_{Y}^{1}(\log S)\right)$, then $U$ is a Shimura curve of Hodge type, and $f: V \rightarrow U$ the corresponding universal family. 
The Lemma 0.7 implies that the Arakelov equality forces $\mathbb{W}=\mathbb{L} \otimes_{\mathbb{C}} \mathbb{T}$ for a unitary system $\mathbb{T}$. In particular, if $S \neq \emptyset$, replacing $Y$ by an étale covering, one can assume that $\mathbb{T}=\mathbb{C}_{U}^{\oplus r}$ and $\mathbb{U}=\mathbb{C}_{U}^{\oplus n-r}$. As in [VZ04] one deduces the next Corollary.

Corollary 0.10. If $S \neq \emptyset$ consists of an even number of points, and if

$$
\operatorname{deg}(\mathcal{A})=\frac{1}{2} \cdot \operatorname{rank}(\mathcal{A}) \cdot \operatorname{deg}\left(\Omega_{Y}^{1}(\log S)\right),
$$

then (replacing $Y$ by an étale covering) $f: X \rightarrow Y$ is isogenous to

$$
E \times_{Y} \cdots \times E_{Y} \times B
$$

where $B$ is an Abelian variety of dimension $n-r$ and where $E \rightarrow Y$ is a modular family of elliptic curves.

The proof of Corollary 0.10 is easy if one assumes that the maximal unitary subsystem of $R^{1} f_{*} \mathbb{C}_{V}$ is defined over $\mathbb{Q}$, and it will be sketched in Section 3 . This condition holds true if $S \neq \emptyset$. For $S=\emptyset$ the proof of Theorem 0.8 in [VZ04] also gives back the known classification of Shimura curves.

As a supplement to 0.8 we will show in Section 3:

Corollary 0.11. If the maximal unitary local subsystem $\mathbb{U}$ of $R^{1} f_{*} \mathbb{C}_{V}$ is trivial, then the family $f: V \rightarrow U$ is rigid. In particular it is defined over $\overline{\mathbb{Q}}$.

Let us consider next families of curves of genus $g \geq 2$, and let us return to the local systems $\mathbb{L}$, defined by logarithmic theta characteristics $\mathcal{L}$. Obviously $\mathbb{L}$ has a strictly maximal Higgs field.

Theorem 0.12 ([Moe2]). Let $f: X \rightarrow Y$ be a semistable family of curves of genus $g \geq 2$, smooth over $U=Y \backslash S$, and $V=f^{-1}(U)$.

1. $R^{1} f_{*} \mathbb{C}_{V}$ contains a rank two sub variation of Hodge structures $\mathbb{L}$ with a strictly maximal Higgs field if and only if $U$ is a Teichmüller curve, and $V \rightarrow U$ the corresponding universal family.

2. Teichmüller curves are defined over number fields.

The definition of a Teichmüller curve will be given in Section 4. Roughly speaking, one considers geodesics in the Teichmüller space, constructed by an $\operatorname{Sl}(2, \mathbb{R})$-action on the real and imaginary part of a given holomorphic differential form. If the quotient by a suitable lattice in $\mathrm{Sl}(2, \mathbb{R})$ is an algebraic curve, it is called a Teichmüller curve in $M_{g}$. As for Shimura curves, we always allow ourselves to replace the lattice by a smaller one, hence the Teichmüller curve by an étale cover. We say that $f: V \rightarrow U$ is the universal family if the morphism $U \rightarrow M_{g}$ is induced by $f$.

It is a striking fact, that in spite of the differential geometric definition of Teichmüller curves, they can be characterized analytically, or even by a numerical property of the Higgs bundle. This has a number of applications 
in the theory of Teichmüller curves (see [Moe2] and [Moe3]), and it allows to construct new examples of such special curves in $M_{g}$.

At the same time, Theorem 0.12 allows to use differential geometric properties of Teichmüller curves to study variations of Hodge structures for families of curves. For example, at the present moment the only known proof of the next Corollary relies on Theorem 0.12, and on differential geometric methods.

Corollary 0.13. In Theorem 0.12 the variation of Hodge structures $R^{1} f_{*} \mathbb{C}_{V}$ can not contain two different sub variation of Hodge structures $\mathbb{L}_{1}$ and $\mathbb{L}_{2}$, both with a strictly maximal Higgs field.

There remains the question whether there are higher rank irreducible sub variations of Hodge structures $\mathbb{V}$ with a strictly maximal Higgs field. By Lemma 0.7 those are of the form $\mathbb{L} \otimes_{\mathbb{C}} \mathbb{T}$, where $\mathbb{T}$ is an irreducible unitary system, at least after replacing $U$ by an étale covering of degree two. As we will see in Section $1 \operatorname{rank}(\mathbb{T}) \geq 2$ implies that $S=\emptyset$, and squeezing the methods used to prove 0.2 a bit more, one can show that this is not possible (see [VZ05]). So putting everything together one obtains:

Corollary 0.14. Let $f: X \rightarrow Y$ be a semistable family of curves of genus $g \geq 2$, smooth over $U=Y \backslash S$. Then $R^{1} f_{*} \mathbb{C}_{V}$ does not contain a sub variation of Hodge structures $\mathbb{V}$ with a strictly maximal Higgs field and with $\operatorname{rank}(\mathbb{V})>$ 2 .

If $S=\emptyset$ then $R^{1} f_{*} \mathbb{C}_{V}$ does not contain any sub variation of Hodge structures $\mathbb{V}$ with a strictly maximal Higgs field.

As we will see in section 4 , the rank two local subsystem $\mathbb{L} \subset R^{1} f_{*} \mathbb{C}_{V}$ can be defined over a number field $K$, i.e. it is of the form $\mathbb{L}_{K} \otimes_{K} \mathbb{C}$ for a local subsystem $\mathbb{L}_{K} \in R^{1} f_{*} K_{V}$, but in general it will not be defined over $\mathbb{Q}$. Replacing $K$ by its Galois hull, for $\sigma \in \operatorname{Gal}(K / \mathbb{Q})$ the conjugate local system $\mathbb{L}^{\sigma}$ can only have a strictly maximal Higgs field, if $\mathbb{L}=\mathbb{L}^{\sigma}$. The defect in the inequality (1) for $\mathbb{L}^{\sigma}$ has been studied in [BM05].

In general things are changing, if one replaces $f: X \rightarrow Y$ by its family of Jacobians. Passing from a family of curves to its Jacobian, one might replace the discriminant locus $S$ by a subset $S^{\prime}$. Singular fibres with a compact Jacobian, for example those with two components meeting in one point, are not contributing to the discriminant locus of the family of Jacobians. If $f: V \rightarrow U$ is the universal family over a Teichmüller curve, then the existence of the local subsystem $\mathbb{L}$ together with the inequality (1) applied to the family of Jacobians, shows that $S^{\prime}=S$, hence that all singular fibres of $f$ have a non-compact Jacobian. One can ask, whether there are any Teichmüller curves $U \subset M_{g}$ in the moduli space of curves of genus $g$ which are Shimura curves in the moduli space $A_{g}$ of Abelian varieties (with a suitable level structure). By [Moe3] the answer is "no" for $g \geq 4$ and for $g=2$, whereas for $g=3$ there exists one example, essentially unique.

On the other hand, by the Corollaries 0.13 and 0.14 we know, that the only curves $U \subset M_{g}$, which are Shimura curves in $A_{g}$ have to be induced by a 
family $f: X \rightarrow Y$ with $R^{1} f_{*} \mathbb{C}_{V}=\mathbb{L} \oplus \mathbb{U}$ with $\operatorname{rank}(\mathbb{L})=2$ and with $\mathbb{U}$ unitary. This implies that $U$ is a Teichmüller curve, and that $U$ is not projective.

\section{Theorem 0.15 .}

a. For $g \geq 2$ the moduli space $M_{g}$ does not contain any compact Shimura curve.

b. $M_{g}$ contains a non-compact Shimura curve $U$ if and only if $g=3$. The Shimura curve $U \in M_{3}$ is a Teichmüller curve, essentially unique.

In this article, we will explain the main ingredients used in the introduction, and we will sketch the proofs of some of the results mentioned above. In Section 1 we explain the Simpson correspondence for variations of Hodge structures over curves, and we will prove Lemma 0.6 and Lemma 0.7 for $k=1$. For $k>1$, Lemma 0.6 will be shown under the additional assumption that $\operatorname{rank}\left(F^{k, 0}\right)=1$.

In Section 2 we prove Theorem 0.3, and we give some hints, how one obtains Corollary 0.14 from Corollary 0.13.

The characterization of Shimura curves will be discussed in Section 3. We prove Theorem 0.8 for families where $R^{1} f_{*} \mathbb{C}_{V}$ itself has a strictly maximal Higgs field, hence we exclude unitary direct factors. We sketch the proofs of Corollaries 0.10 and 0.11 and we also reproduce the proof of Theorem 0.9 from [Moe3].

Section 4 gives an introduction to the theory of Teichmüller curves. In particular we sketch the proof of Theorem 0.12 and of the Corollary 0.13.

Finally the non-existence of Teichmüller curves in $M_{g}$, for $g=2$ and $g \geq 4$ and the proof of Theorem 0.15, b), will be discussed in Section 5 .

\section{Higgs bundles over curves and Arakelov inequalities}

We will frequently use C. Simpson's correspondence between polystable logarithmic Higgs bundles of degree zero and representations of the fundamental group $\pi_{1}(U, *)$. Recall that a logarithmic Higgs bundle is a locally free sheaf $E$ on $Y$ together with an $\mathcal{O}_{Y}$ linear morphism $\theta: E \rightarrow E \otimes \Omega_{Y}^{1}(\log S)$ with $\theta \wedge \theta=0$. The usual definitions of stability (and semistability) for locally free sheaves extend to Higgs bundles, by requiring that

$$
\mu(F)=\frac{\operatorname{deg}(F)}{\operatorname{rank}(F)}<\mu(E)=\frac{\operatorname{deg}(E)}{\operatorname{rank}(E)}
$$

(or $\mu(F) \leq \mu(E)$ ) for all subsheaves $F$ with $\theta(F) \subset F \otimes \Omega_{Y}^{1}(\log S)$.

Theorem 1.1 (C. Simpson [Si90]). There exists a natural equivalence between the category of direct sums of stable filtered regular Higgs bundles of degree zero, and of direct sums of stable filtered local systems of degree zero. 
We will not recall the definition of a "filtered regular" Higgs bundle [Si90, page 717], and just remark that for a Higgs bundle corresponding to a local system $\mathbb{V}$ with unipotent monodromy around the points in $S$ the filtration is trivial, and automatically $\operatorname{deg}(\mathbb{V})=0$.

In general it is impossible to describe the Higgs bundle $(E, \theta)$ explicitly in terms of the corresponding local system $\mathbb{V}$, with two exceptions:

Example 1.2. If $\mathbb{T}$ is a unitary local system on $U$ with unipotent monodromy operators in $s \in S$, then the corresponding Higgs bundle is the Deligne extension $N$ of $N_{0}=\mathbb{T} \otimes \mathcal{O}_{U}$ and the Higgs field $\theta$ is the zero map.

The second example already occurred in the Introduction.

Example 1.3. Let $\mathbb{V}$ be a polarized $\mathbb{C}$ variation of Hodge structures of weight $k$ and with unipotent local monodromy operators. The $\mathcal{F}$-filtration on $F_{0}=$ $\mathbb{V} \otimes_{\mathbb{C}} \mathcal{O}_{U}$ extends to a locally splitting filtration on the Deligne extension $F$

$$
\mathcal{F}^{k+1} \subset \mathcal{F}^{k} \subset \cdots \subset \mathcal{F}^{0} .
$$

We will usually assume that $\mathcal{F}^{k+1}=0$ and $\mathcal{F}^{0}=F$. The Griffiths transversality condition for the Gauß-Manin connection $\nabla$ says that

$$
\nabla\left(\mathcal{F}^{p}\right) \subset \mathcal{F}^{p-1} \otimes \Omega_{Y}^{1}(\log S),
$$

and hence $\nabla$ induces a $\mathcal{O}_{Y}$ linear map

$$
\theta_{p, k-p}: F^{p, k-p}=\mathcal{F}^{p} / \mathcal{F}^{p+1} \longrightarrow F^{p-1, k-p+1}=\mathcal{F}^{p-1} / \mathcal{F}^{p} \otimes \Omega_{T}^{1}(\log S) .
$$

So

$$
\left(F=\bigoplus_{p} F^{p, k-p}, \theta=\bigoplus \theta_{p, k-p}\right)
$$

is a Higgs bundle, and it is the image of $\mathbb{V}$ under the Simpson correspondence in Theorem 1.1.

Properties 1.4. Let $\mathbb{V}$ be a direct sum of irreducible $\mathbb{C}$ local system and let $(F, \theta)$ be the corresponding Higgs bundle.

1. $F=\bigoplus_{p+q=k} F^{p, q}$ with $\theta\left(F^{p, q}\right) \subset F^{p-1, q+1} \otimes \Omega_{Y}^{1}(\log S)$ if and only if $\mathbb{V}$ is a local system underlying a complex polarized variation of Hodge structures.

2. Under the equivalent conditions in 1 ) let $\mathbb{T}$ be a local subsystem of $\mathbb{V}$ with Higgs bundle

$$
\left(N=\bigoplus_{p} N^{p, k-p}, 0\right) \subset\left(F=\bigoplus_{p} F^{p, k-p}, \theta=\bigoplus \theta_{p, k-p}\right) .
$$

Then $\mathbb{T}$ is unitary. 
Proof. The condition on $(F, \theta)$ in 1$)$ is just saying, that it is a system of Hodge bundles, as defined in [Si88]. So the first part follows from [Si88] and [Si90].

Let $\Theta(N, h)$ denote the curvature of the Hodge metric $h$ on $F$ restricted to $N$, then by [Gr84], chapter II we have

$$
\Theta\left(N,\left.h\right|_{N}\right)=-\theta_{N} \wedge \bar{\theta}_{N}-\bar{\theta}_{N} \wedge \theta_{N}=0 .
$$

This means that $\left.h\right|_{N}$ is a flat metric. Hence, $\mathbb{T}$ is a unitary local system.

The Simpson correspondence does not say anything about the field of definition for $\mathbb{V}$. Here we say that $\mathbb{V}$ is defined over a subfield $K$ of $\mathbb{C}$ if there is a $K$-local system $\mathbb{V}_{K}$ with $\mathbb{V}=\mathbb{V}_{K} \otimes_{K} \mathbb{C}$. In different terms, for $\mu=\operatorname{rank}(\mathbb{V})$ the representation

$$
\gamma_{\mathbb{V}}: \pi_{0}(U, *) \longrightarrow \operatorname{Gl}(\mu, \mathbb{C})
$$

is conjugate to one factoring like

$$
\gamma_{\mathbb{V}}: \pi_{0}(U, *) \longrightarrow \operatorname{Gl}(\mu, K) \longrightarrow \operatorname{Gl}(\mu, \mathbb{C}) .
$$

If $\mathbb{V}$ is defined over $K$, and if $\sigma: K \rightarrow K^{\prime}$ is an isomorphism, we will write $\mathbb{V}_{K}^{\sigma}$ for the local system defined by

$$
\gamma_{\mathbb{V}}: \pi_{0}(U, *) \longrightarrow \mathrm{Gl}(\mu, K) \stackrel{\sigma}{\longrightarrow} \mathrm{Gl}\left(\mu, K^{\prime}\right),
$$

and $\mathbb{V}^{\sigma}=\mathbb{V}_{K}^{\sigma} \otimes_{K^{\prime}} \mathbb{C}$.

By [De71] (for $K=\mathbb{Q}$ ) and by [De87] the category of polarized $K$ variations of Hodge structures is semisimple. By [De87, Proposition 1.13] one has:

Lemma 1.5. A local system $\mathbb{V}$, underlying a polarized variation of Hodge structures, decomposes as

$$
\mathbb{V}=\bigoplus_{i=1}^{r}\left(\mathbb{V}_{i} \otimes W_{i}\right)
$$

where $\mathbb{V}_{i}$ are pairwise non-isomorphic irreducible $\mathbb{C}$-local systems and $W_{i}$ are non-zero $\mathbb{C}$-vector spaces.

Moreover the $\mathbb{V}_{i}$ and the $W_{i}$ carry polarized variations of Hodge structures, whose tensor product and sum gives back the Hodge structure on $\mathbb{V}$. The Hodge structure on the $\mathbb{V}_{i}$ (and $W_{i}$ ) is unique up to a shift of the bigrading.

Suppose that $\mathbb{W}$ is a local system defined over a number field $L$. The local system $\mathbb{W}_{L}$ is given by a representation $\rho: \pi_{1}(U, *) \rightarrow \operatorname{Gl}\left(W_{L}\right)$ for the fibre $W_{L}$ of $\mathbb{W}_{L}$ over the base point $*$.

Fixing a positive integer $r<\mu$ let $\mathcal{G}(r, \mathbb{W})$ denote the set of all rank$\mathrm{r}$ local subsystems of $\mathbb{W}$ and let $\operatorname{Grass}\left(r, W_{L}\right)$ be the Grassmann variety of $r$-dimensional subspaces. Then $\mathcal{G}(r, \mathbb{W})$ is the subvariety of

$$
\operatorname{Grass}\left(r, W_{L}\right) \times_{\operatorname{Spec}(L)} \operatorname{Spec}(\mathbb{C})
$$


consisting of the $\pi_{1}(U, *)$-invariant points. In particular, it is a projective variety defined over $L$. A $K$-valued point of $\mathcal{G}(r, \mathbb{W})$ corresponds to a local subsystem of $\mathbb{W}_{K}=\mathbb{W}_{L} \otimes_{L} K$. One obtains the following well known property.

Lemma 1.6. If $[\mathbb{V}] \in \mathcal{G}(r, \mathbb{W})$ is an isolated point, then $\mathbb{V}$ is defined over $\overline{\mathbb{Q}}$.

Lemma 1.7. Let $\mathbb{W}$ be a polarized variation of Hodge structures defined over $L$, and let $\mathbb{V} \subset \mathbb{W}$ be an irreducible local subsystem of rank $r$ defined over $\mathbb{C}$,. Then $\mathbb{V}$ can be deformed to a local subsystem $\mathbb{V}_{t} \subset \mathbb{W}$, which is isomorphic to $\mathbb{V}$ and which is defined over a finite extension of $L$.

Proof. By Lemma $1.5 \mathbb{W}$ is completely reducible over $\mathbb{C}$. Hence we have a decomposition $\mathbb{W}=\mathbb{V} \oplus \mathbb{V}^{\prime}$.

The space $\mathcal{G}(r, \mathbb{W})$ of rank $r$ local subsystems of $\mathbb{W}$ is defined over $L$ and the subset

$$
\left\{\mathbb{V}_{t} \in \mathcal{G}(r, \mathbb{W}) ; \text { the composite } \mathbb{V}_{t} \subset \mathbb{V} \oplus \mathbb{V}^{\prime} \stackrel{p r_{1}}{\longrightarrow} \mathbb{V} \text { is non zero }\right\}
$$

forms a Zariski open subset. So there exists some $\mathbb{V}_{t}$ in this subset, which is defined over some finite extension of $L$. Since $p: \mathbb{V}_{t} \rightarrow \mathbb{V}$ is non zero, since $\operatorname{rank}\left(\mathbb{V}_{t}\right)=\operatorname{rank}(\mathbb{V})$, and since $\mathbb{V}$ is irreducible, $p$ is an isomorphism.

The Lemmata 1.6 and 1.7 are just the starting points to show, that certain local subsystems of $R^{k} f_{*} \mathbb{C}_{V}$ are defined over number fields (see [VZ04]). Let us give a typical example:

Lemma 1.8. Let $\mathbb{W}$ be defined over a (real) number field, and let $\mathbb{W}=\mathbb{V} \oplus \mathbb{U}$ be a decomposition such that the Higgs field of $\mathbb{V}$ is maximal, and such that $\mathbb{U}$ is unitary.

1. Then $\mathbb{V}$ and $\mathbb{U}$ are defined over a (real) number field, as well as the decomposition.

2. If $S \neq \emptyset$, then $\mathbb{U}$ is defined over $\mathbb{Q}$ and it trivializes over an étale covering of $Y$.

Proof. Consider a family $\mathbb{V}_{t}$ of local subsystems of $\mathbb{W}$ for $t$ in a small disk $\Delta$, and with $\mathbb{V}_{0}=\mathbb{V}$. Since the Higgs field of $\mathbb{V}_{0}$ is maximal, one may assume that the one of $\mathbb{V}_{t}$ is maximal for all $t \in \Delta$. Then the projection $\mathbb{V}_{t} \rightarrow \mathbb{U}$ must be zero. Otherwise, the complete reducibility of local systems coming from variations of Hodge structures in 1.5 implies that $\mathbb{V}_{t}$ and $\mathbb{U}$ have a common factor, obviously a contradiction.

So $\mathbb{V}$ is rigid as a local subsystem, hence defined over $\overline{\mathbb{Q}}$. The same argument works for $\mathbb{U}$ instead of $\mathbb{V}$.

If $\mathbb{W}$ is defined over $\mathbb{R}$, then $\overline{\mathbb{V}} \rightarrow \mathbb{U}$ again has to be the zero map. So $\mathbb{V}$ and $\mathbb{U}$ have to be defined over $\mathbb{R}$.

Assume now that $S \neq \emptyset$. By (1) we know that the decomposition is defined over a number field. For the local monodromy operators "unitary and 
unipotent" implies that the nilpotent part of the monodromy is zero. This is invariant under conjugation by $\sigma \in \operatorname{Gal}(\overline{\mathbb{Q}} / \mathbb{Q})$, and $\mathbb{U}^{\sigma}=\mathbb{U}$.

Finally, since $\mathbb{U}$ is a local subsystem of a variation of Hodge structures, it carries a $\mathbb{Z}$-structure. Then the image of the corresponding monodromy representation is finite.

Lemma 1.5 together with Theorem 1.1 implies that the Higgs bundle $(F, \theta)$ of a variation of Hodge structures $\mathbb{V}$ is polystable (as a Higgs bundle) and of degree zero. As a first application one obtains Kollár's decomposition of the sheaf $F^{k, 0}$, mentioned in the introduction.

Lemma 1.9. Let $\left(F=F^{k, 0} \oplus \cdots \oplus F^{0, k}, \theta\right)$ be the logarithmic Higgs bundle of a variation of Hodge structures $\mathbb{V}$ with unipotent local monodromies. Then $F^{k, 0}=\mathcal{A} \oplus \mathcal{B}$ with $\mathcal{A}$ ample and with $\mathcal{B}$ flat.

Proof. Replacing $\mathbb{V}$ by one of its irreducible direct factors, one has to show that $F^{k, 0}$ is ample, provided that $\theta \neq 0$.

A quotient sheaf $\mathcal{N}$ of $F^{k, 0}$ gives rise to a quotient Higgs bundle $(\mathcal{N}, 0)$ of $(F, \theta)$, hence 1.1 implies that $\operatorname{deg}(\mathcal{N}) \geq 0$. If $\operatorname{deg}(\mathcal{N})=0$, then it corresponds to a local subsystem, which is excluded by the irreducibility of $\mathbb{V}$. Hence all quotients of $F^{k, 0}$ have strictly positive degree, which implies that $F^{k, 0}$ is ample.

Let us consider for a moment the case $k=1$ and $\mathbb{V}=R^{1} f_{*} \mathbb{C}_{V}$. Then $(\mathcal{B} \oplus$ $\left.\mathcal{B}^{\vee}, 0\right)$ is a sub Higgs bundle, hence by Lemma 1.4, 2), it corresponds to a unitary subbundle of $\mathbb{V}$. The latter is defined over $\mathbb{Q}$ and by Lemma 1.8, (1), this decomposition is defined over $\overline{\mathbb{Q}} \cap \mathbb{R}$.

The Arakelov inequality stated in Lemma 0.6 follows from the polystability of the Higgs bundles (see [VZ03] and [VZ05]). We will indicate the proof just in two simple cases, the one of weight one variations of Hodge structures, and the one where the $(k, 0)$ part is one dimensional.

Proof of Lemma 0.6 for weight one. Let $\mathcal{A} \subset F^{1,0}$ be a subsheaf, and let $\mathcal{C} \otimes \Omega_{Y}^{1}(\log S)$ be its image under $\theta_{1,0}$. Then $\mathcal{A} \oplus \mathcal{C}$ is a Higgs subbundle of $F^{1,0} \oplus F^{0,1}$, and 1.1 implies that $\operatorname{deg}(\mathcal{A})+\operatorname{deg}(\mathcal{C}) \leq 0$. Since $\left(\operatorname{ker}\left(\left.\theta\right|_{\mathcal{A}}\right), 0\right)$ is a sub Higgs bundle, one has

$$
\begin{aligned}
\operatorname{deg}(\mathcal{A}) \leq \operatorname{deg}(\mathcal{C})+ & \operatorname{rank}(\mathcal{C}) \cdot \operatorname{deg}\left(\Omega_{Y}^{1}(\log S)\right) \\
\leq \operatorname{deg}(\mathcal{C})+ & \operatorname{rank}(\mathcal{A}) \cdot \operatorname{deg}\left(\Omega_{Y}^{1}(\log S)\right) \\
& \leq-\operatorname{deg}(\mathcal{A})+\operatorname{rank}(\mathcal{A}) \cdot \operatorname{deg}\left(\Omega_{Y}^{1}(\log S)\right)
\end{aligned}
$$

and

$$
\frac{\operatorname{deg}(\mathcal{A})}{\operatorname{rank}(\mathcal{A})} \leq \frac{1}{2} \operatorname{deg}\left(\Omega_{Y}^{1}(\log S)\right) .
$$

For $\mathcal{A}=F^{1,0}$ one obtains the inequality (1) in 0.6. 
If (1) is an equality, all the inequalities in (3) are equalities. This implies that $\operatorname{rank}(\mathcal{A})=\operatorname{rank}(\mathcal{C})$, hence that $\left.\theta\right|_{\mathcal{A}}$ is injective. Furthermore $\operatorname{deg}(\mathcal{A})+$ $\operatorname{deg}(\mathcal{C})=0$, hence $\mathcal{A} \oplus \mathcal{C}$ corresponds to a sub-local system of $\mathbb{V}$ with a maximal Higgs field.

In fact we have shown a bit more. Assume that (1) in Lemma 0.6 is an equality. If $\mathbb{V}$ is irreducible

$$
\frac{\operatorname{deg}(\mathcal{A})}{\operatorname{rank}(\mathcal{A})}<\frac{1}{2} \operatorname{deg}\left(\Omega_{Y}^{1}(\log S)\right)=\frac{\operatorname{deg}\left(F^{1,0}\right)}{g_{0}},
$$

except for $\mathcal{A}=F^{1,0}$. Hence $F^{1,0}$ is stable. By duality one obtains the same for $F^{0,1}$.

If $\mathbb{V}$ is not irreducible, applying this argument to all the direct factors of $\mathbb{V}$ one finds:

Lemma 1.10. If $\left(F^{1,0} \oplus F^{0,1}, \theta\right)$ is the logarithmic Higgs bundle of a variation of Hodge structures $\mathbb{V}$ with unipotent local monodromies and with a strictly maximal Higgs field, then the sheaves $F^{1,0}$ and $F^{0,1}$ are polystable and the Higgs field $\theta: F^{1,0} \rightarrow F^{0,1} \otimes \Omega_{Y}^{1}(\log S)$ is a morphism between polystable sheaves of the same slope.

Although in this survey we only consider families over curves, let us make one remark about families over a higher dimensional basis. One can hope that the polystability of the Hodge bundles $F^{1,0}$ and $F^{0,1}$ is again enforced by a numerical condition on the degrees with respect to $\omega_{Y}(S)$, and that it forces $U=Y \backslash S$ to be a Shimura variety. Some first results in this direction have been obtained in [VZ05b].

Proof of Lemma 0.7 for $k=1$. By assumption $\# S$ is even, so we can choose a logarithmic theta characteristic $\mathcal{L}$ and an isomorphism

$$
\tau: \mathcal{L} \rightarrow \mathcal{L}^{-1} \otimes \Omega_{Y}^{1}(\log S),
$$

giving us the $\mathbb{C}$-variation of Hodge structures $\mathbb{L}$. By Lemma 1.10, the sheaf $\mathcal{T}=F^{1,0} \otimes \mathcal{L}^{-1}$ is polystable of degree zero, hence isomorphic to $\mathbb{T} \otimes \mathcal{O}_{Y}$ for a unitary local system $\mathbb{T}$. The isomorphism

$$
\mathcal{T} \otimes \mathcal{L}=F^{1,0} \stackrel{\theta}{\simeq} F^{0,1} \otimes \Omega_{Y}^{1}(\log S) \underset{\simeq}{\longrightarrow} F^{0,1} \otimes \mathcal{L}^{2}
$$

induces an isomorphism $\phi: \mathcal{T} \otimes \mathcal{L}^{-1} \stackrel{\simeq}{\longrightarrow} F^{0,1}$, such that $\theta=\phi \circ\left(\operatorname{id}_{\mathcal{T}} \otimes \tau\right)$. Hence the Higgs bundles $\left(F^{1,0} \oplus F^{0,1}, \theta\right)$ and $\left(\mathcal{T} \otimes\left(\mathcal{L} \oplus \mathcal{L}^{-1}\right), \operatorname{id}_{\mathcal{T}} \otimes \tau\right)$ are isomorphic, and $\mathbb{V} \simeq \mathbb{T} \otimes_{\mathbb{C}} \mathbb{L}$.

Proof of Lemma 0.6 under the additional assumption $\operatorname{rank}\left(F^{k, 0}\right)=1$. For later use, let us consider a slightly more general situation:

$\mathbb{V}$ is a variation of polarized complex Hodge structures and with unipotent 
local monodromy in $s \in S$, and with logarithmic Higgs bundle $\left(\bigoplus F^{k, 0}, \theta\right)$. Let $\mathcal{H} \subset F^{k, 0}$ be an invertible subsheaf, and let $E \subset F$ be the sub Higgs bundle generated by $\mathcal{H}$. Writing $T_{Y}(-\log S)$ for the dual of $\Omega_{Y}^{1}(\log S)$, for some $q_{0}$

$$
E^{k-q, q}=F^{k-q, q} \cap E=\left\{\begin{array}{ll}
\mathcal{H} \otimes T_{Y}(-\log S)^{q} & \text { for } q \leq q_{0} \\
0 & \text { for } q>q_{0} .
\end{array} .\right.
$$

Theorem 1.1 implies that

$$
\operatorname{deg}(E)=\left(q_{0}+1\right) \cdot \operatorname{deg}(\mathcal{H})-\frac{q_{0} \cdot\left(q_{0}+1\right)}{2} \operatorname{deg}\left(\Omega_{Y}^{1}(\log S)\right) \leq 0,
$$

hence

$$
\operatorname{deg}(\mathcal{H}) \leq \frac{q_{0}}{2} \operatorname{deg}\left(\Omega_{Y}^{1}(\log S)\right) \leq \frac{k}{2} \operatorname{deg}\left(\Omega_{Y}^{1}(\log S)\right) .
$$

In Lemma 0.6 we choose $\mathcal{H}=F^{k, 0}$, and we obtain the Arakelov inequality. If this is an equality, then $q_{0}=k$ and $E$ corresponds to a local subsystem, by construction with a strictly maximal Higgs field.

\section{Coverings and bounds for subsheaves of the direct images}

Let us recall from [VZ05] the proof of the following proposition, as a first application of the theory of Higgs bundles.

Proposition 2.1. Let $f: X \rightarrow Y$ be a semistable family of $n$-folds over a curve $Y$, and smooth over $U=Y \backslash S$. If $Y=\mathbb{P}^{1}$ assume that $\# S \geq 2$, and consider for $\nu \geq 1$ an invertible subsheaf $\mathcal{H}$ of $f_{*} \omega_{X / Y}^{\nu}$. Then

$$
\operatorname{deg} \mathcal{H} \leq \frac{n \cdot \nu}{2} \cdot \operatorname{deg}\left(\Omega_{Y}^{1}(\log S)\right) .
$$

Before sketching the proof of the proposition, let us show that it implies Theorem 0.3.

Proof of Theorem 0.3. Since either $g(Y) \geq 1$, or $Y=\mathbb{P}^{1}$ and $\# S \geq 2$, one can always find a covering $Y^{\prime} \rightarrow Y$, which is unramified over $U$ and which has a prescribed ramification order in $s \in S$. Since $f$ is semistable, the inequality in Theorem 0.3 is compatible with replacing $Y$ by $Y^{\prime}$ and $f$ by a desingularization of the pullback family.

For $r=\operatorname{rank}\left(f_{*} \omega_{X / Y}^{\nu}\right)$ consider the family $f^{(r)}: X^{(r)} \rightarrow Y$, obtained as a desingularization of the total space of the $r$ fold product

$$
X^{r}=X \times_{Y} \cdots \times_{Y} X \longrightarrow Y .
$$

Remark that $X^{r}$ is normal, Gorenstein with at most rational singularities. Hence flat base change implies that 


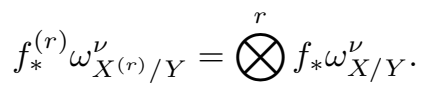

The family is perhaps not semistable, but replacing $Y$ by a covering sufficiently ramified in $s \in S$ the pullback will have this property (This step is not needed if one replaces in 2.1 "semistable" by "unipotent local monodromy"). So Proposition 2.1 implies for $\operatorname{det}\left(f_{*} \omega_{X / Y}^{\nu}\right) \subset f_{*}^{(r)} \omega_{X^{(r)} / Y}^{\nu}$ that

$$
\operatorname{deg}\left(f_{*} \omega_{X / Y}^{\nu}\right) \leq \frac{\operatorname{rank}\left(f_{*} \omega_{X / Y}^{\nu}\right) \cdot n \cdot \nu}{2} \cdot \operatorname{deg}\left(\Omega_{Y}^{1}(\log S)\right) .
$$

Proof of 2.1. For $\nu=1$ this is the inequality (4), obtained at the end of the last section. Hence we will only consider the case $\nu>1$ in the sequel.

Assume that $\operatorname{deg}(\mathcal{H})>\frac{n \cdot \nu}{2} \cdot \operatorname{deg}\left(\Omega_{Y}^{1}(\log S)\right)$. Replacing $Y$ by a finite covering, étale over $U$, one may assume that

$$
\operatorname{deg}(\mathcal{H})=\nu \cdot \rho>\frac{n \cdot \nu}{2} \cdot \operatorname{deg}\left(\Omega_{Y}^{1}(\log S)\right) .
$$

Let $P$ be an effective divisor on $Y$ of degree $\rho$. Then $\mathcal{H} \otimes \mathcal{O}_{Y}(-\nu \cdot P)$ is in $\operatorname{Pic}_{0}(Y)$, hence divisible. So for some invertible sheaf $\mathcal{N}$ of degree zero, for $F=f^{*} P$ and for $\mathcal{L}=\omega_{X / Y} \otimes f^{*} \mathcal{N} \otimes \mathcal{O}_{X}(-F)$ the sheaf $\mathcal{L}^{\nu}$ has a non-zero section $\sigma$. It gives rise to a cyclic covering of $X$ whose desingularization will be denoted by $\hat{W}$ (see [EV92], for example). Then for some divisor $\hat{T}$ the morphism $\hat{h}: \hat{W} \rightarrow Y$ will be smooth over $Y \backslash \hat{T}$, but not semistable. Choose $Y^{\prime}$ to be a covering, sufficiently ramified, such that the pullback family has a semistable model over $Y^{\prime}$.

Next choose $W^{\prime}$ to be a $\mathbb{Z} / \nu$ equivariant desingularization of $\hat{W} \times_{Y} Y^{\prime}$, and $Z$ to be a desingularization of the quotient. Finally let $W$ be the normalization of $Z$ in the function field of $\hat{W} \times_{Y} Y^{\prime}$. So we have a diagram

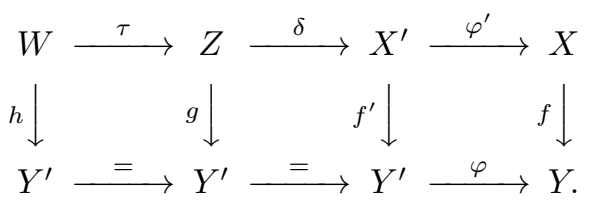

The $\nu$-th power of the sheaf $\mathcal{M}=\delta^{*} \varphi^{\prime *} \mathcal{L}$ has the section $\sigma^{\prime}=\delta^{*} \varphi^{\prime *}(\sigma)$. The sum of its zero locus and the singular fibres will become a normal crossing divisor after a further blowing up. Replacing $Y^{\prime}$ by a larger covering, one may assume that $Z \rightarrow Y^{\prime}$ is semistable, and that $Z$ and $D$ satisfy the assumption iii) stated below.

For a suitable choice of $T$ one has the following conditions:

i. $X^{\prime}=X \times_{Y} Y^{\prime}$, and $\tau: W \rightarrow Z$ is the finite covering obtained by taking the $\nu$-th root out of $\sigma^{\prime} \in H^{0}\left(Z, \mathcal{M}^{\nu}\right)$. 
ii. $g$ and $h$ are both smooth over $Y^{\prime} \backslash T$ for a divisor $T$ on $Y^{\prime}$ containing $\varphi^{-1}(S)$. Moreover $g$ is semistable and the local monodromy of $R^{n} h_{*} \mathbb{C}_{W \backslash h^{-1}(T)}$ in $t \in T$ are unipotent.

iii. $\delta$ is a modification, and $Z \rightarrow Y^{\prime}$ is semistable. Writing $\Delta^{\prime}=g^{*} T$ and $D$ for the zero divisor of $\sigma^{\prime}$ on $Z$, the divisor $\Delta^{\prime}+D$ has normal crossing and $D_{\text {red }} \rightarrow Y^{\prime}$ is étale over $Y^{\prime} \backslash T$.

iv. $\delta_{*}\left(\omega_{Z / Y^{\prime}} \otimes \mathcal{M}^{-1}\right)=\varphi^{*}\left(\mathcal{N}^{-1} \otimes \mathcal{O}_{Y}(P)\right)$,

In fact, since $f: X \rightarrow Y$ is semistable, $X^{\prime}$ has at most rational double points. Then

$$
\delta_{*}\left(\omega_{Z / Y^{\prime}} \otimes \delta^{*} \varphi^{\prime *} \omega_{X / Y}^{-1}\right)=\delta_{*}\left(\omega_{Z / Y^{\prime}} \otimes \delta^{*} \omega_{X^{\prime} / Y^{\prime}}^{-1}\right)=\delta_{*} \omega_{Z / X^{\prime}}=\mathcal{O}_{X^{\prime}},
$$

which implies iv). The properties i), ii) and iii) hold by construction.

$W$ might be singular, but the sheaf $\Omega_{W / Y^{\prime}}^{p}\left(\log \tau^{*} \Delta^{\prime}\right)=\tau^{*} \Omega_{Z / Y^{\prime}}^{1}\left(\log \Delta^{\prime}\right)$ is locally free and compatible with desingularizations. The Galois group $\mathbb{Z} / \nu$ acts on the direct image sheaves $\tau_{*} \Omega_{W / Y^{\prime}}^{p}\left(\log \tau^{*} \Delta^{\prime}\right)$. As in [EV92] or [VZ05, Section 3] one has the following description of the sheaf of eigenspaces.

Claim. Let $\Gamma^{\prime}$ be the sum over all components of $D$, whose multiplicity is not divisible by $\nu$. Then the sheaf

$$
\Omega_{Z / Y^{\prime}}^{p}\left(\log \left(\Gamma^{\prime}+\Delta^{\prime}\right)\right) \otimes \mathcal{M}^{-1} \otimes \mathcal{O}_{Z}\left(\left[\frac{D}{\nu}\right]\right),
$$

is a direct factor of $\tau_{*} \Omega_{W / Y^{\prime}}^{p}\left(\log \tau^{*} \Delta^{\prime}\right)$. Moreover the $\mathbb{Z} / \nu$ action on $W$ induces a $\mathbb{Z} / \nu$ action on

$$
\mathbb{W}=R^{n} h_{*} \mathbb{C}_{W \backslash \tau^{-1} \Delta^{\prime}}
$$

and on its Higgs bundle. One has a decomposition of $\mathbb{W}$ in a direct sum of sub variations of Hodge structures, given by the eigenspaces for this action, and the Higgs bundle of one of them is of the form $G=\bigoplus_{q=0}^{n} G^{n-q, q}$ for

$$
G^{p, q}=R^{q} g_{*}\left(\Omega_{Z / Y^{\prime}}^{p}\left(\log \left(\Gamma^{\prime}+\Delta^{\prime}\right)\right) \otimes \mathcal{M}^{-1} \otimes \mathcal{O}_{Z}\left(\left[\frac{D}{\nu}\right]\right)\right) .
$$

The Higgs field $\theta_{p, q}: G^{p, q} \rightarrow G^{p-1, q+1} \otimes \Omega_{Y^{\prime}}^{1}(\log T)$ is induced by the edge morphisms of the exact sequence

$$
\begin{aligned}
0 \longrightarrow \Omega_{Z / Y^{\prime}}^{p-1}\left(\log \left(\Gamma^{\prime}+\Delta^{\prime}\right)\right) \otimes g^{*} \Omega_{Y^{\prime}}^{1}(\log T) & \\
\longrightarrow \Omega_{Z}^{p}\left(\log \left(\Gamma^{\prime}+\Delta^{\prime}\right)\right) \longrightarrow \Omega_{Z / Y^{\prime}}^{p}\left(\log \left(\Gamma^{\prime}+\Delta^{\prime}\right)\right) & \longrightarrow 0
\end{aligned}
$$

tensorized with $\mathcal{M}^{-1} \otimes \mathcal{O}_{Z}\left(\left[\frac{D}{\nu}\right]\right)$.

The sheaf

$$
G^{n, 0}=g_{*}\left(\Omega_{Z / Y^{\prime}}^{n}\left(\log \left(\Gamma^{\prime}+\Delta^{\prime}\right)\right) \otimes \mathcal{M}^{-1} \mathcal{O}_{Z}\left(\left[\frac{D}{\nu}\right]\right)\right)
$$


contains the invertible sheaf

$$
\mathcal{H}=g_{*}\left(\Omega_{Z / Y^{\prime}}^{n}\left(\log \Delta^{\prime}\right) \otimes \mathcal{M}^{-1}\right)=g_{*}\left(\omega_{Z / Y^{\prime}} \otimes \mathcal{M}^{-1}\right)=\varphi^{*}\left(\mathcal{N} \otimes \mathcal{O}_{Y}(P)\right) .
$$

of degree $\operatorname{deg}(\varphi) \cdot \rho$.

Claim. $\mathcal{H}$ generates a sub Higgs bundle

$$
\left(H=\bigoplus_{q=0}^{n} H^{n-q, q},\left.\theta\right|_{H}\right)
$$

of $(G, \theta)$ where

$$
H^{n-q-1, q+1}=\operatorname{Im}\left(\left.\theta\right|_{H^{n-q, q}}: H^{n-q, q} \rightarrow G^{n-q+1, q+1} \otimes \omega_{Y^{\prime}}(T)\right) \otimes \varphi^{*} \omega_{Y}(S)^{-1} .
$$

For some $q_{0}$ the sheaf $H^{n-q, q}$ is invertible of degree

$$
\operatorname{deg}(\varphi) \cdot\left(\rho-q \cdot \operatorname{deg}\left(\Omega_{Y}^{1}(\log S)\right)\right)
$$

for $q \leq q_{0}$ and zero for $q>q_{0}$.

Proof. Writing $\Delta=f^{*}(S)$ consider the tautological exact sequences

$$
0 \rightarrow \Omega_{X / Y}^{p-1}(\log \Delta) \otimes f^{*} \Omega_{Y}^{1}(\log S) \longrightarrow \Omega_{X}^{p}(\log \Delta) \longrightarrow \Omega_{X / Y}^{p}(\log \Delta) \rightarrow 0,
$$

tensorized with

$$
\omega_{X / Y}^{-1}=\left(\Omega_{X / Y}^{n}(\log \Delta)\right)^{-1} .
$$

Taking the edge morphisms one obtains a Higgs bundle starting with the $(n, 0)$ part $\mathcal{O}_{Y}$. The sub Higgs bundle generated by $\mathcal{O}_{Y}$ has $\Omega_{Y}^{1}(\log S)^{-q}$ in degree $(n-q, q)$. Tensorizing with $\mathcal{N}^{-1} \otimes \mathcal{O}_{Y}(P)$ one obtains a Higgs bundle $H_{0}$ with

$$
H_{0}^{n-q, q}=\mathcal{N}^{-1} \otimes \mathcal{O}_{Y}(p) \otimes \omega_{Y}(S)^{-q} .
$$

On the other hand, the pullback of the exact sequence (7) to $Z$ is a subsequence of

$$
0 \rightarrow \Omega_{Z / Y^{\prime}}^{p-1}\left(\log \Delta^{\prime}\right) \otimes g^{*} \Omega_{Y^{\prime}}^{1}(\log T) \rightarrow \Omega_{Z}^{p}\left(\log \Delta^{\prime}\right) \rightarrow \Omega_{Z / Y^{\prime}}^{p}\left(\log \Delta^{\prime}\right) \rightarrow 0,
$$

hence of the sequence (6), as well. So the Higgs field of $\varphi^{*} H_{0}$ is induced by the edge morphism of the exact sequence (6), tensorized with

$$
\varphi^{\prime *}\left(\omega_{X / Y}^{-1} \otimes f^{*}\left(\mathcal{N}^{-1} \otimes \mathcal{O}_{Y}(P)\right)\right),
$$

or with the larger sheaf $\mathcal{M}^{-1} \otimes \mathcal{O}_{Z}\left(\left[\frac{D}{\nu}\right]\right)$.

One obtains a morphism of Higgs bundles $\varphi^{*} H_{0} \rightarrow G$. By definition

$$
\varphi^{*} H_{0}^{n, 0}=\varphi^{*}\left(\mathcal{N}^{-1} \otimes \mathcal{O}_{Y}(P)\right)=H^{n, 0} \stackrel{\subset}{\longrightarrow} G^{n, 0},
$$

and $H$ is the image of $\varphi^{*} H_{0}$ in $G$. 
The last claim implies that the degree of $H$ is

$$
\begin{aligned}
& \operatorname{deg}(\varphi) \cdot\left(\left(q_{0}+1\right) \cdot \rho-\sum_{q=0}^{q_{0}} q \cdot \operatorname{deg}\left(\Omega_{Y}^{1}(\log S)\right)\right)= \\
& \operatorname{deg}(\varphi) \cdot\left(\left(q_{0}+1\right) \cdot \rho-\frac{q_{0} \cdot\left(q_{0}+1\right)}{2} \cdot \operatorname{deg}\left(\Omega_{Y}^{1}(\log S)\right)\right)> \\
& \operatorname{deg}(\varphi) \cdot\left(\frac{\left(q_{0}+1\right) \cdot n}{2}-\frac{q_{0} \cdot\left(q_{0}+1\right)}{2}\right) \cdot \operatorname{deg}\left(\Omega_{Y}^{1}(\log S)\right) \geq 0
\end{aligned}
$$

contradicting the polystability of $(G, \theta)$.

Corollary 2.2. [VZ05, Theorem 6] Consider a semistable family $f: X \rightarrow$ $Y$ of $n$-folds over a curve $Y$, smooth over $U=Y \backslash S$. Let $\mathbb{V}$ be a $\mathbb{C}$-sub variation of Hodge structures in $R^{n} f_{*} \mathbb{C}_{V}$, with $(n, 0)$ part $F^{n, 0}$ and with a strictly maximal Higgs field. Then the rational map $\pi: X \rightarrow \mathbb{P}\left(F^{n, 0}\right)$, defined by $f^{*} F^{n, 0} \rightarrow \omega_{X / Y}$, can not have a non-isotrivial image. In particular $\pi$ can not be birational.

Sketch of the proof. By Lemma 0.7 one may assume that $F^{n, 0}=\mathcal{L}^{n} \otimes \mathcal{T}$ for a logarithmic theta characteristic $\mathcal{L}$ and for a unitary bundle $\mathcal{T}$ on $Y$. Let $\mathcal{B}_{\nu}$ denote the image of the multiplication map

$$
\mathfrak{m}: S^{\nu}\left(F^{n, 0}\right) \longrightarrow f_{*} \omega_{X / Y}^{\nu}
$$

If the image of $\pi$ is non-isotrivial, the method used to prove [Vi95, Theorem 4.33] shows that $\operatorname{deg}\left(\mathcal{B}_{\nu} \otimes \mathcal{L}^{-\nu \cdot n}\right)$ has to be larger than zero, for some $\nu \gg 1$. For $r=\operatorname{rank}\left(\mathcal{B}_{\nu}\right)$ one obtains an inclusion

$$
\operatorname{det}\left(\mathcal{B}_{\nu}\right) \longrightarrow \bigotimes^{r} f_{*} \omega_{X / Y}^{\nu}
$$

Since

$$
\operatorname{deg}\left(\mathcal{B}_{\nu}\right)>r \cdot \nu \cdot n \cdot \operatorname{deg}(\mathcal{L})=\frac{r \cdot n \cdot \nu}{2} \cdot \operatorname{deg}\left(\omega_{X / Y}^{\nu}\right)
$$

this contradicts Proposition 2.1, applied to a desingularization of the $r$ fold fibre product $X \times_{Y} \cdots \times_{Y} X \rightarrow Y$.

Sketch of the proof of: "0.13 $\Longrightarrow 0.14$ ". We assume again that $\mathbb{V}$ has a maximal Higgs field, that $\operatorname{rank}(\mathbb{V})>2$ and we write $F^{1,0}$ for the first Hodge bundle.

By Corollary 2.2 it only remains to consider the case where the image $W$ of $\pi$ is isotrivial. For $n=1$ one shows (replacing $Y$ by an étale covering) that $W \rightarrow Y$ is either of the form $C \times Y \rightarrow Y$, for some curve $C$ of positive genus, or it is a $\mathbb{P}^{1}$ bundle. In the first case, it is easy to see, that $\mathbb{P}\left(F^{1,0}\right)$ is trivial, which implies by Lemma 0.7 that $\mathbb{V}$ is the direct sum of copies of $\mathbb{L}$ contradicting Corollary 0.13.

A similar argument works in the second case, and we refer to [VZ05] for the details. 


\section{Maximal Higgs fields and Shimura curves}

In this section $f: X \rightarrow Y$ is a family of $g$-dimensional Abelian varieties. We assume as usual that the family is semistable, or slightly weaker, that the local monodromies of $R^{1} f_{*} \mathbb{C}_{V}$ in $s \in S$ are all unipotent. We will sketch the proof of Theorem 0.8 under the quite restrictive assumption

Assumption 3.1. $\mathbb{V}=R^{1} f_{*} \mathbb{C}_{V}$ has no unitary part.

Of course we will also assume that the Higgs field of $R^{1} f_{*} \mathbb{C}_{V}$ is strictly maximal. So the Assumption 3.1 will exclude all non rigid families. The proof of Theorem 0.8 without it is unfortunately much more difficult.

For $S \neq \emptyset$ the Assumption 3.1 is not really a restriction. If $R^{1} f_{*} \mathbb{C}_{V}=$ $\mathbb{V} \oplus \mathbb{U}$ where $\mathbb{V}$ has a strictly maximal Higgs field, and where $\mathbb{U}$ is unitary, the assumption on $S$ implies by Lemma 1.8, (2), that (replacing $U$ by an étale covering) this decomposition is defined over $\mathbb{Q}$ and that $\mathbb{U}$ is trivial. So $f: X \rightarrow Y$ is isogenous to the product of a family $f^{\prime}: X^{\prime} \rightarrow Y$, with $\mathbb{V}$ as variation of Hodge structures, and a constant Abelian variety. In this case, the Assumption 3.1 holds if one replaces $f: X \rightarrow Y$ by its "moving part" $f^{\prime}: X^{\prime} \rightarrow Y$.

By Lemma 0.7 there is a decomposition $R^{1} f_{*} \mathbb{C}_{V}=\mathbb{L} \otimes_{\mathbb{C}} \mathbb{T}$, and by the choice of $\mathbb{L}$ one has $\operatorname{det}(\mathbb{L})=\mathbb{C}$, hence $\operatorname{det}(\mathbb{T})=\mathbb{C}$, as well. Again one can show, that such a decomposition exists with $\mathbb{L}$ and $\mathbb{T}$ defined over real number fields. Here one applies the arguments used in the proof of Lemma 1.8 to the local system $\mathbb{E n d}(\mathbb{L} \otimes \mathbb{T})$.

One has a description of $g$-fold wedge products of tensor products (see [FH91], for example) in terms of partitions $\lambda=\left\{\lambda_{1}, \ldots, \lambda_{\nu}\right\}$ of $g$. By definition $\lambda_{1}, \ldots, \lambda_{\nu}$ are natural numbers with $g=\lambda_{1}+\cdots+\lambda_{\nu}$. The partition $\lambda$ defines a Young diagram and a Schur functor $\mathbb{S}_{\lambda}$. Some standard elementary calculations show that:

\section{Lemma 3.2.}

a. If $k$ is odd, then for some partitions $\lambda_{c}$,

$$
\bigwedge^{k}(\mathbb{L} \otimes \mathbb{T})=\bigoplus_{c=0}^{\frac{k-1}{2}} S^{2 c+1}(\mathbb{L}) \otimes \mathbb{S}_{\lambda_{2 c}}(\mathbb{T})
$$

b. If $k$ is even, then for some partitions $\lambda_{c}$,

$$
\bigwedge(\mathbb{L} \otimes \mathbb{T})=S^{k}(\mathbb{L}) \oplus \mathbb{S}_{\{2, \ldots, 2\}}(\mathbb{T}) \oplus \bigoplus_{c=1}^{\frac{k}{2}-1} S^{2 c}(\mathbb{L}) \otimes \mathbb{S}_{\lambda_{2 c}}(\mathbb{T})
$$

\section{Lemma 3.3.}

a. If $k$ is odd,

$$
H^{0}\left(Y, \bigwedge^{k}(\mathbb{L} \otimes \mathbb{T})\right)=0
$$


b. If $k$ is even, say $k=2 c$,

$$
H^{0}\left(Y, \bigwedge^{k}(\mathbb{L} \otimes \mathbb{T})\right)=H^{0}\left(Y, \bigwedge^{k}(\mathbb{L} \otimes \mathbb{T})\right)^{c, c}=H^{0}\left(Y, \mathbb{S}_{\{2, \ldots, 2\}}(\mathbb{T})\right)
$$

Proof. $S^{\ell}(\mathbb{L})$ has a strictly maximal Higgs field for $\ell>0$, whereas for all partitions $\lambda^{\prime}$ the variation of Hodge structures $\mathbb{S}_{\lambda^{\prime}}(\mathbb{T})$ is again pure of bidegree 0,0 . The local system $S^{\ell}(\mathbb{L}) \otimes \mathbb{S}_{\lambda^{\prime}}(\mathbb{T})$ has again a strictly maximal Higgs field. A global section gives a trivial local subsystem, hence a Higgs subbundle of the form $\left(\mathcal{O}_{Y}, 0\right)$, contradicting the strict maximality of the Higgs field. So $S^{\ell}(\mathbb{L}) \otimes \mathbb{S}_{\lambda^{\prime}}(\mathbb{T})$ has no global sections.

Hence $\bigwedge^{k}(\mathbb{L} \otimes \mathbb{T})$ can only have global sections for $k$ even. In this case, the global sections lie in

$$
\operatorname{det}(\mathbb{L})^{c} \otimes \mathbb{S}_{\{2, \ldots, 2\}}(\mathbb{T})=\mathbb{S}_{\{2, \ldots, 2\}}(\mathbb{T}) .
$$

Let $F$ be an Abelian variety and let $Q$ be the polarization, i.e. a nondegenerate antisymmetric form on $H^{1}(F, \mathbb{Q})$. The Hodge group $\operatorname{Hg}(F)$ is defined in [Mu66] (see also [Mu69]) as the smallest $\mathbb{Q}$-algebraic subgroup of $\operatorname{Sp}\left(H^{1}(F, \mathbb{Q}), Q\right)$, whose extension to $\mathbb{R}$ contains the complex structure

$$
u: S^{1} \longrightarrow \operatorname{Sp}\left(H^{1}(F, \mathbb{R}), Q\right) .
$$

Here $z$ acts on $(p, q)$ cycles by multiplication with $z^{p} \cdot \bar{z}^{q}$.

In a similar way, one defines the Mumford-Tate group $\mathrm{MT}(F)$. The complex structure $u$ extends to a morphism of real algebraic groups

$$
h: \operatorname{Res}_{\mathbb{C} / \mathbb{R}} \mathbb{G}_{m} \longrightarrow \operatorname{Gl}\left(H^{1}(F, \mathbb{R})\right),
$$

and $\operatorname{MT}(F)$ is the smallest $\mathbb{Q}$-algebraic subgroup of $\operatorname{Gl}\left(H^{1}(F, \mathbb{Q})\right.$ ), whose extension to $\mathbb{R}$ contains the image (see [De72], [De82] [Moo98] and [Sch96]). Let us recall some of its properties, stated in [Moo98] and [De72] with the necessary references. $\mathrm{MT}(F)$ is a reductive group, and it preserves the intersection form $Q$ up to scalar multiplication.

$\operatorname{MT}(F)$ is also the largest $\mathbb{Q}$-algebraic subgroup of $\operatorname{Gl}\left(H^{1}(F, \mathbb{Q})\right.$ ), which leaves all Hodge cycles of $F \times \cdots \times F$ invariant, hence all elements

$$
\eta \in H^{2 p}(F \times \cdots \times F, \mathbb{Q})^{p, p}=\left[\bigwedge^{2 p}\left(H^{1}(F, \mathbb{Q}) \oplus \cdots \oplus H^{1}(F, \mathbb{Q})\right)\right]^{p, p} .
$$

For a smooth family of Abelian varieties $f: V \rightarrow U$ there exist the union $\Sigma$ of countably many proper closed subvarieties of $Y$ such that $\operatorname{MT}\left(f^{-1}(y)\right)$ is independent of $y$ for $y \in U \backslash \Sigma$ (see [De72], [Moo98] or [Sch96]). Let us fix such a very general point $y \in U \backslash \Sigma$ in the sequel and $F=f^{-1}(y)$. One defines $\operatorname{MT}\left(R^{1} f_{*} \mathbb{Q}_{V}\right)$ to be the Mumford-Tate group of $F$. 
Consider Hodge cycles $\eta$ on $F$ which remain Hodge cycles under parallel transform. Then $\operatorname{MT}\left(R^{1} f_{*} \mathbb{Q}_{V}\right)$ is the largest $\mathbb{Q}$-subgroup of $\operatorname{Gl}\left(H^{1}(F, \mathbb{Q})\right)$ which leaves all those Hodge cycles invariant ([De82, §7] or [Sch96, 2.2]). For an algebraic group $G$ the derived group $G^{\text {der }}$ is the subgroup generated by all commutators, or equivalently the kernel of the homomorphism $G \rightarrow G^{\text {ab }}$ for $G$ to the maximal Abelian quotient.

Let $\mathrm{Mon}^{0}$ be the algebraic monodromy group, i.e. the connected component of the Zariski closure of the image of the monodromy representation. Let us recall two results from [De82] and [An92] (see [Moo98, 1.4]).

\section{Proposition 3.4.}

a. $\mathrm{Mon}^{0}$ is a normal subgroup of the derived subgroup $\mathrm{MT}(F)^{\mathrm{der}}$ of $\mathrm{MT}(F)$.

b. If for some $y^{\prime} \in Y$ the fibre $f^{-1}\left(y^{\prime}\right)$ has complex multiplication, then $\operatorname{Mon}^{0}=\operatorname{MT}\left(R^{1} f_{*} \mathbb{Q}_{V}\right)^{\text {der }}$.

Remark however, that up to now we do not know anything about the existence of points with complex multiplication for the families considered in Theorem 0.8. So instead of $3.4, \mathrm{~b}$ ), we will use:

Proposition 3.5. Let $f: X \rightarrow Y$ be a family of $g$-dimensional Abelian varieties. Assume that the local monodromies of $R^{1} f_{*} \mathbb{C}_{V}$ are all unipotent and that its Higgs field is strictly maximal. Then $\operatorname{Mon}^{0}=\operatorname{MT}\left(R^{1} f_{*} \mathbb{Q}_{V}\right)^{\text {der }}$.

Proof. Let us write $\mathrm{MT}=\mathrm{MT}\left(R^{1} f_{*} \mathbb{Q}_{V}\right)$ and let us fix a very general fibre $F$ of $f$. By [Si92, 4.4] $\mathrm{Mon}^{0}$ is reductive. By [De82, 3.1 (c)] it is sufficient to show that each tensor

$$
\eta \in \bigwedge^{k}\left(H^{1}(F, \mathbb{Q}) \oplus \cdots \oplus H^{1}(F, \mathbb{Q})\right)=H^{k}(F \times \cdots \times F, \mathbb{Q})
$$

which is invariant under $\mathrm{Mon}^{0}$ is also invariant under $\mathrm{MT}^{\mathrm{der}}$. By abuse of notations, let us replace $F \times \cdots \times F$ by $F$.

A section $\eta \in H^{k}(F, \mathbb{Q}) \backslash\{0\}$ gives rise to a global section

$$
\eta \in H^{0}\left(U, \bigwedge^{k} \mathbb{L} \otimes_{\mathbb{C}} \mathbb{T}\right)
$$

By Lemma 3.3, a) $k$ must be even, and by part b) the section $\eta$ is pure of bidegree $\left(\frac{k}{2}, \frac{k}{2}\right)$. So $\eta$ is a Hodge cycle, and by definition it is invariant under MT.

Mumford defines in [Mu66] a Shimura variety $\mathcal{X}(\mathrm{Hg}, u)$ of Hodge type as a moduli scheme of Abelian varieties (with a suitable level structure) whose Hodge group is contained in Hg. In [Mu69] he gives an explicit construction. $\mathcal{X}(\mathrm{Hg}, u)$ is the image of

$$
\Phi: \operatorname{Hg}_{\mathbb{R}} \longrightarrow \operatorname{Sp}\left(H^{1}(F, \mathbb{Q}), Q\right)_{\mathbb{R}} /\left(\begin{array}{c}
\text { centralizer of the } \\
\text { complex structure } u
\end{array}\right)=\mathcal{H}_{g}
$$


divided by an arithmetic subgroup $\Gamma \subset \mathrm{Hg}$. The kernel of $\Phi$ is a maximal compact subgroup.

The monodromy group for $\mathcal{X}(\mathrm{Hg}, u)$ is contained in $\mathrm{Hg}$, hence equal to

$$
\mathrm{Hg}^{\text {der }}=\mathrm{MT}^{\text {der }} \cap \mathrm{Hg} \text {. }
$$

Since

$$
\left(\mathrm{MT}^{\mathrm{der}} \times \mathrm{MT}\right) / \mathrm{MT}^{\mathrm{der}}
$$

is isogenous to MT, we may replace in Mumford's construction $\mathrm{Hg}$ by $\mathrm{Hg}^{\text {der }}$, and the dimension of $\mathcal{X}(\mathrm{Hg}, u)$ is the dimension of $\Phi\left(\mathrm{Hg}^{\mathrm{der}}\right)$.

Proof of Theorem 0.8 under the Assumption 3.1.

The decomposition $R^{1} f_{*} \mathbb{C}_{V}=\mathbb{L} \otimes_{\mathbb{C}} \mathbb{T}$ implies that the representation defining the local system has values in the product of $\mathrm{Sl}(2, \mathbb{R})$ with a compact group. In particular, the dimension of $\mathcal{X}(\mathrm{Hg}, u)$ is one, and the induced morphism $\varphi^{\prime}: U \rightarrow \mathcal{X}(\mathrm{Hg}, u)$ is dominant.

Consider the composite $\varphi$

$$
U \stackrel{\varphi^{\prime}}{\longrightarrow} \mathcal{X}(\mathrm{Hg}, u) \longrightarrow \mathcal{A}_{g},
$$

where $\mathcal{A}_{g}$ denotes a fine moduli scheme of Abelian varieties, with a suitable level structure. If $\left(F^{1,0} \oplus F^{0,1}, \theta\right)$ denotes the Higgs bundle of $R^{1} f_{*} \mathbb{C}_{V}$ the pullback of tangent sheaf of $\mathcal{A}_{g}$ is given by $\varphi^{*} T_{\mathcal{A}_{g}}=\left.S^{2}\left(F^{0,1}\right)\right|_{U}$, and $T_{U} \rightarrow$ $\varphi^{*} T_{\mathcal{A}_{g}}$ is the map

$$
\left.T_{U} \longrightarrow S^{2}\left(\left.F^{0,1}\right|_{U}\right) \longrightarrow\left(F^{0,1} \otimes F^{1,0 \vee}\right)\right|_{U}=\mathcal{L}_{0}^{-1} \otimes \mathcal{T}_{0} \otimes \mathcal{L}_{0}^{-1} \otimes \mathcal{T}_{0}^{\vee}
$$

induced by $\theta$. Here $\mathcal{L}_{0}$ is the restriction to $U$ of the logarithmic theta characteristic, and $\mathcal{T}_{0}=\mathbb{T} \otimes \mathcal{O}_{U}$. So $T_{U}$ is just the subsheaf $\mathcal{L}_{0}^{-2} \otimes \mathcal{O}_{U}$, where $\mathcal{O}_{U} \subset \mathcal{H o m}\left(\mathcal{T}_{0}, \mathcal{T}_{0}\right)$ is given by the homotheties. In particular $T_{U}$ is a direct factor of $\varphi^{*} T_{\mathcal{A}_{g}}$, and $\varphi$ and $\varphi^{\prime}$ are both étale.

On the proof of Corollary 0.10. By Lemma 1.8, (2), the assumption $S \neq \emptyset$ implies that the unitary system $\mathbb{T}$ becomes trivial over some étale covering, as well as the maximal unitary sub-local system $\mathbb{U}$ in $R^{1} f_{*} \mathbb{C}_{V}$. So we may assume, that this holds true on $Y$ itself. So $f: X \rightarrow Y$ is isogenous to a constant Abelian variety, corresponding to the unitary subsystem $\mathbb{U}$ and the moving part $f^{\prime}: X^{\prime} \rightarrow Y$. By abuse of notations, we will assume that $f=f^{\prime}$, hence that $\mathbb{U}=0$.

Assume first that the general fibre $F$ of $f$ is simple, hence $\mathbb{U}=0$, and $\mathbb{T}=\mathbb{C}^{\oplus g}$.

Sections in $H^{0}\left(U, \mathbb{E}\right.$ nd $\left.\left(R^{1} f_{*} \mathbb{C}_{V}\right)\right)$ correspond to trivial Higgs subbundles of $\mathcal{E} n d\left(\left(\mathcal{L} \oplus \mathcal{L}^{-1}\right)^{\oplus g}\right)$. The rang of this bundle is $4 \cdot g^{2}$ and a rank $3 \cdot g^{2}$ subsystem has a strictly maximal Higgs field. The rank of the largest sub Higgs bundle with a trivial Higgs field is $g^{2}$, and it is concentrated in bidegree $(0,0)$. Since the corresponding local sub system of $\mathbb{E n d}\left(R^{1} f_{*} \mathbb{C}_{V}\right)$ is unitary, Lemma 1.8 
implies that it is defined over $\mathbb{Q}$ and that it trivializes over an étale covering. So we may assume that

$$
H^{0}\left(U, \mathbb{E n d}\left(R^{1} f_{*} \mathbb{Q}_{V}\right)\right)=H^{0}\left(U, \mathbb{E n d}\left(R^{1} f_{*} \mathbb{Q}_{V}\right)\right)^{0,0}=\mathbb{Q}^{g^{2}},
$$

hence that $\operatorname{End}(F)_{\mathbb{Q}}=\mathbb{Q}^{g^{2}}$. Now take your favorite textbook on Abelian varieties and look for simple ones, with $\operatorname{End}(F)_{\mathbb{Q}}=\mathbb{Q}^{g^{2}}$, and with a positive dimensional non-compact moduli space. The only examples you will find are families of elliptic curves. So $f: X \rightarrow Y$ is a modular family of elliptic curves, completely determined by the local system $\mathbb{L}$.

If $F$ is not simple, apply the argument indicated above to each simple factor of the family.

Sketch of the proof of Corollary 0.11.

The description $R^{1} f_{*} \mathbb{C}_{V}=\mathbb{L} \otimes \mathbb{T}$ implies that

$$
H^{0}\left(U, \mathbb{E n d}\left(R^{1} f_{*} \mathbb{C}_{V}\right)\right)=H^{0}\left(U, \mathbb{E n d}\left(R^{1} f_{*} \mathbb{C}_{V}\right)\right)^{0,0} .
$$

Then the rigidity follows from [Fa83].

Let $X_{\mathrm{Hg}}$ be the $\mathrm{Hg}(\mathbb{R})^{+}$-conjugacy class in

$$
\operatorname{Hom}_{\text {alg.grp } / \mathbb{R}}\left(\operatorname{Res}_{\mathbb{C} / \mathbb{R}} \mathbb{G}_{m}, \operatorname{Hg}_{\mathbb{R}}\right)
$$

containing $u \circ\left(\operatorname{Res}_{\mathbb{C} / \mathbb{R}} \mathbb{G}_{m} \rightarrow S^{1}\right)$. Here + denotes the topological connected component. For the reader's convenience we note that $\left(\mathrm{Hg}, X_{\mathrm{Hg}}\right)$ is a Shimura datum in the sense of [De79, 2.1.1]:

$\mathrm{Hg}$ is reductive and [De79] Proposition 1.1.14 derives the axioms (2.1.1.1) and (2.1.1.2) from the fact that $u$ defines a complex structure compatible with the polarization. The axiom (2.1.1.3), i.e. the non-existence of a $\mathbb{Q}$-factor in $\mathrm{Hg}^{\text {ad }}$ onto which $h$ projects trivially, follows from $\mathrm{Hg}$ being the smallest $\mathbb{Q}$-subgroup of $\operatorname{Sp}\left(H^{1}(F, \mathbb{Q}), Q\right)$ containing $u$.

Sketch of the proof of Theorem 0.9. Since Hg is reductive we may split the representation $\mathrm{Hg} \rightarrow \operatorname{Sp}\left(H^{1}(F, \mathbb{Q}), Q\right)$ into a direct sum of irreducible representations. We may split off unitary representations over $\mathbb{Q}$ ([Kol87] Proposition 4.11).

Claim. For each of the remaining irreducible representations there is an isogeny $i: \mathrm{Sl}_{2}(\mathbb{R}) \times K \rightarrow \mathrm{Hg}_{\mathbb{R}}$, where $K$ is a compact group, such that the composition of $i$ with $\mathrm{Hg}_{\mathbb{R}} \rightarrow \operatorname{Sp}\left(H^{1}(F, \mathbb{Q}), Q\right)_{\mathbb{R}}$ is the tensor product of a representation of $\mathrm{Sl}_{2}(\mathbb{R})$ of weight one by a representation of $K$.

Assuming the Claim, consider a maximal compact subgroup $K_{1}$ of $\mathrm{Sl}_{2}(\mathbb{R}) \times K$ that maps to the centralizer of $u$ under

$$
\mathrm{Sl}_{2}(\mathbb{R}) \times K \longrightarrow \operatorname{Sp}\left(H^{1}(F, \mathbb{Q}), Q\right)_{\mathbb{R}}
$$

The double quotient 


$$
\mathcal{X}^{\prime}=\Gamma^{\prime} \backslash\left(\mathrm{Sl}_{2}(\mathbb{R}) \times K\right) / K_{1}
$$

is an unramified cover of $\mathcal{X}(\mathrm{Hg}, U)$. Since a strictly maximal Higgs field is characterized by the Arakelov equality we may as well prove that the pullback variation of Hodge structures has a strictly maximal Higgs field. Since the fundamental group of $\mathcal{X}^{\prime}$ acts via $\Gamma^{\prime}$ this follows immediately from the claim and Lemma 2.1 in [VZ04].

Proof of the Claim. We first analyze $\mathrm{Hg}^{\text {ad }}$ and the $\mathrm{Hg}^{\mathrm{der}}(\mathbb{R})$-conjugacy class $X_{\mathrm{Hg}}^{\text {ad }}$ of maps $\operatorname{Res}_{\mathbb{C} / \mathbb{R}} \mathbb{G}_{m} \rightarrow \mathrm{Hg}^{\text {ad }}$ containing $\left(\mathrm{Hg} \rightarrow \mathrm{Hg}^{\text {ad }}\right) \circ u$. Note that $X_{\mathrm{Hg}}^{\text {ad }}$ is a connected component of $X_{\mathrm{Hg}}$.

Each $\mathbb{Q}$-factor of $\mathrm{Hg}^{\text {ad }}$ onto which $h$ projects non-trivially contributes to the dimension of $\mathcal{H}(\mathrm{Hg}, u)$. Since we deal with Shimura curves $\mathrm{Hg}^{\text {ad }}$ is $\mathbb{Q}$-simple by (2.1.1.3). Let

$$
\operatorname{Hg}_{\mathbb{R}}^{\mathrm{ad}}=\prod_{i \in I} G_{i}
$$

be its decomposition into simple factors. Then $X_{\mathrm{Hg}}^{\text {ad }}=\prod X_{i}$ for $X_{i}$ a $G_{i}(\mathbb{R})$ conjugacy class of maps $\operatorname{Res}_{\mathbb{C} / \mathbb{R}} \mathbb{G}_{m} \rightarrow G_{i}$. For the same reason, only one of the simple factors, say $G_{1}$, of $\mathrm{Hg}_{\mathbb{R}}^{\text {ad }}=\prod_{i \in I} G_{i}$ is non-compact. The possible complexifications $\left(G_{1}\right)_{\mathbb{C}}$ are classified by Dynkin diagrams. The property 'Shimura curve', i.e. dimension one, implies that $G_{1} \cong \operatorname{PSl}(2, \mathbb{R})$.

Now we determine the possible representations. The universal cover $\widetilde{G_{1}} \rightarrow$ $G_{1}$ factors though

$$
G:=\operatorname{Ker}\left(\mathrm{Hg} \rightarrow \prod_{i \in I \backslash\{1\}} G_{i}\right)^{0} .
$$

We apply [De79] Section 1.3 to

$$
\left(G_{1}, X_{\mathrm{Hg}}\right) \leftarrow\left(G, X_{\mathrm{Hg}}\right) \rightarrow(\mathrm{Sp}, \mathcal{H}) .
$$

Since a finite-dimensional representation of $G_{1}$ factors through $\mathrm{Sl}(2, \mathbb{R})$, we conclude that $G \cong S l(2, \mathbb{R})$. Moreover, such a representation corresponds to a fundamental weight, hence of weight one. Now we let $\widetilde{K}$ be the universal cover of $\prod_{i \in I \backslash\{1\}} G_{i}$. Since $\mathrm{Hg} \rightarrow \mathrm{Hg}^{\text {ad }}$ is an isogeny, there is a lift of the universal cover $\widetilde{K} \rightarrow \mathrm{Hg}$. This lift factors though a quotient $K$ of $\widetilde{K}$ such that the natural map $\mathrm{Sl}(2, \mathbb{R}) \times K \rightarrow \mathrm{Hg}_{\mathbb{R}}$ is an isogeny.

Since we assumed the representation $\operatorname{Hg}_{\mathbb{R}} \rightarrow \operatorname{Sp}\left(H^{1}(F, \mathbb{Q}), Q\right)_{\mathbb{R}}$ to be irreducible, also

$$
\rho: \operatorname{Sl}(2, \mathbb{R}) \times K \rightarrow \operatorname{Hg}_{\mathbb{R}} \rightarrow \operatorname{Sp}\left(H^{1}(F, \mathbb{Q}), Q\right)_{\mathbb{R}}
$$

is irreducible. Let $W \subset H^{1}(F, \mathbb{R})$ be an irreducible (necessarily weight one) representation of $\mathrm{Sl}(2, \mathbb{R}) \times\{$ id $\}$. Since $K$ is reductive, hence its representations are semisimple, $\rho$ is the tensor product of $W$ and the representation $\operatorname{Hom}_{\mathrm{SI}(2, \mathbb{R}) \times\{\operatorname{id}\}}\left(W, H^{1}(F, \mathbb{R})\right)$ of $K$. This completes the proof of the claim. 


\section{Teichmüller curves and their variation of Hodge structures}

We start with the assumptions of Theorem 0.12: Let $f: X \rightarrow Y$ be a semistable family of curves of genus $g \geq 2$, smooth over $U=Y \backslash S$ and let $V=f^{-1}(U)$. Suppose that $R^{1} f_{*} \mathbb{C}_{V}$ contains a sub variation of Hodge structures $\mathbb{L}$ of rank two with a strictly maximal Higgs field. Let us for the moment admit the following:

Lemma 4.1. $\mathbb{L}$ is defined over $\mathbb{R}$

By Theorem 0.2 the universal covering of $U$ is the upper half plane $\mathcal{H}$. Let $\widetilde{\varphi}: \mathcal{H} \rightarrow \mathcal{H}$ the period map for $\mathbb{L}$. The strictly maximality of the Higgs field is now equivalent to $\widetilde{\varphi}$ being an isomorphism (compare [VZ04] Lemma 2.1). In different terms $\widetilde{\varphi}$ is an isometry for the Kobayashi metric on $\mathcal{H}$. The choice of a Teichmüller marking (see below) on one of the smooth fibres of $f$ defines a lift of the moduli map $m: U \rightarrow M_{g}$ to Teichmüller space. We denote this lift by $\widetilde{m}: \mathcal{H} \rightarrow T_{g}$ and we let $j: T_{g} \rightarrow \mathcal{H}_{g}$ the natural map to the Siegel half space, which associates to the curve its Jacobian and to the Teichmüller marking the choice of a symplectic basis. Finally we let $p_{11}: \mathcal{H}_{g} \rightarrow \mathcal{H}$ the projection of the matrix in $\mathcal{H}_{g}$ to its $(1,1)$-entry. If we have chosen the symplectic basis $B$ suitably, i.e. such that the first pair in $B$ spans the fibres of $\mathbb{L}$, then

$$
\widetilde{\varphi}=p_{11} \circ j \circ \widetilde{m} .
$$

Since the composite map $\widetilde{\varphi}$ is a Kobayashi isometry, also $\widetilde{m}$ is an isometry, if we provide the image with the restriction of the Kobayashi metric on $T_{g}$. By a theorem of Royden the Kobayashi metric on $T_{g}$ coincides with the Teichmüller metric. To sum up:

The image of $U$ in $M_{g}$ is an algebraic curve, whose lift in $T_{g}$ is a complex geodesic for the Teichmüller metric. These objects are called Teichmüller curves. We have thus proved one implication of Theorem 0.12.1.

Before showing the converse implication we need some background on geodesics for the Teichmüller metric. Details may be found in [IT92].

One of the equivalent definitions of Teichmüller space is the following. Let $T_{g}$ be the space of pairs $(R, h)$ of a Riemann surface plus a quasi-conformal mapping $h: R_{0} \rightarrow R$ up to isotopy (the Teichmüller marking) from a 'reference surface' $R_{0}$ to $R$. By Teichmüller's theorem the isotopy class of $h$ contains a unique representative with minimal maximal dilatation. Its Beltrami coefficient (a $(-1,1)$-form) $\mu=h_{\bar{z}} / h_{z}$ is of the form $\mu=t|q| / q$ for some quadratic differential $q \in \Gamma\left(R_{0},\left(\Omega_{R_{0}}^{1}\right)^{\otimes 2}\right)$ and $|t|<1$. Conversely, one can solve the Beltrami equation for any $\mu$ of with $|\mu|_{\infty}<1$. In particular for each $\mu=t|q| / q$ there is a quasi-conformal map $h: R_{0} \rightarrow R$ for some Riemann surface $R$ whose Beltrami coefficient is $\mu$.

A quadratic differential $q$ on a Riemann surface $R_{0}$ determines an atlas of $R$ minus the zero set of $q_{0}$ such that the transition functions are translations 
maybe composed by multiplication with \pm 1 : The charts $\psi_{i}: U_{i} \rightarrow \mathbb{C}$ are given by integrating by a square root of $q$ that exists locally. The condition for $h$ to have minimal maximal dilatation may be rephrased by saying that $h$ is affine in the charts $\psi_{i}$ for some $q$ on $R_{0}$ and some ('terminal') quadratic differential on $R$. The Teichmüller geodesic from id to $h$ is given by the conformal maps with Beltrami coefficient $c t|q| / q$ for $c \in[0,1]$. Thus for each $q$, the set

$$
\left\{\mu_{q}(t):=t|q| / q,|t|<1\right\}
$$

defines totally geodesic subset of $T_{g}$.

We briefly recall the construction of the complex structure on $T_{g}$ that makes $T_{g} \rightarrow M_{g}$ holomorphic. Given $(R, h)$ or rather its Beltrami coefficient $\mu$ on $R_{0}$, pull it back to universal covering $\mathcal{H}$ of $R_{0}$ and extend it by zero to obtain a Beltrami coefficient $\tilde{\mu}$ on $\mathbb{C}$. Solving the Beltrami equation for $\mu$ gives a quasi-conformal mapping $h: \mathbb{C} \rightarrow \mathbb{C}$, conformal on the lower half plane $\mathcal{H}^{*}$. The Schwarzian derivative of $\left.\tilde{h}\right|_{\mathcal{H}^{*}}$ is a quadratic differential on $\mathcal{H}^{*}$. Moreover, since $\mu$ was pulled back from $R=\mathcal{H} / \Gamma_{R}$, the Schwarzian derivative descends from $\mathcal{H}^{*}$ to a quadratic differential

$$
q_{\mu}^{*} \in H^{0}\left(\mathcal{H}^{*} / \Gamma_{R},\left(\Omega_{\mathcal{H}^{*} / \Gamma_{R}}^{1}\right)^{\otimes 2}\right) .
$$

The map $\mu \mapsto q_{\mu}^{*}$ factors through Teichmüller space and the complex structure on $H^{0}\left(\mathcal{H}^{*} / \Gamma_{R},\left(\Omega_{\mathcal{H}^{*} / \Gamma_{R}}^{1}\right)^{\otimes 2}\right)$ is the one we want.

The map $\mu \mapsto q_{\mu}^{*}$ has a section locally around 0 (due to Bers) given by $q^{*}(z) \mapsto-2 \operatorname{Im}(z) q^{*}(\bar{z})$. This implies that a neighborhood of 0 of

$$
\left\{\mu_{q}(t),|t|<1\right\}
$$

is a holomorphic submanifold of $T_{g}$. Since the complex structure on $T_{g}$ is independent of the base point, all of $\left\{\mu_{q}(t),|t|<1\right\}$ is a holomorphic submanifold.

To sum up: For a fixed Riemann surface $R_{0}$ and $q \in \Gamma\left(R_{0},\left(\Omega_{R_{0}}^{1}\right)^{\otimes 2}\right)$ fixed, the set $t|q| / q,|t|<1$ is a totally geodesic and holomorphic submanifold of $T_{g}$, a Teichmüller disc.

In order to characterize which (few!) Teichmüller discs descend to Teichmüller curves in $M_{g}$ we provide another description of a Teichmüller disc. There is a natural $\mathrm{Sl}(2, \mathbb{R})$-action on the bundle of triples $(R, \varphi, q)$ over $T_{g}$. Post-compose local charts $\psi_{i}$ of $R$ with $A \in \mathrm{Sl}(2, \mathbb{R})$ acting on $\mathbb{C} \cong \mathbb{R}^{2}$. Since overlapping charts differ only by translations and multiplication by \pm 1 , the compositions

$$
\widetilde{\psi}_{i}: U_{i} \longrightarrow \mathbb{C} \cong \mathbb{R}^{2} \stackrel{A}{\longrightarrow} \mathbb{R}^{2} \cong \mathbb{C}
$$

determine a new complex structure on $R$. We let this new Riemann surface be $A \cdot R$. There is a unique quadratic differential $A \cdot q$ on $A \cdot R$ whose integration charts are $\widetilde{\psi}_{i}$.

The action of $\mathrm{SO}(2, \mathbb{R})$ does not change the complex structure of $R$. Hence the orbit $\operatorname{Sl}(2, \mathbb{R}) \cdot(R, \varphi, q)$ projects to a disc in $T_{g}$. If we lift 


$$
\begin{aligned}
\mathcal{H} & \rightarrow \operatorname{Sl}(2, \mathbb{R}) \\
\tau & \mapsto\left(\begin{array}{l}
1 \operatorname{Re} \tau \\
0 \operatorname{Im} \tau
\end{array}\right)
\end{aligned}
$$

one verifies that the Beltrami coefficient of the composition $\widetilde{\psi}_{i}$ is $\frac{i-\tau}{i+\tau} \frac{|q|}{q}$. Hence the image of the $\mathrm{Sl}(2, \mathbb{R})$-orbit is a Teichmüller disc.

Proposition 4.2. A Teichmüller disc descends to a Teichmüller curve, if and only if the setwise stabilizer of the disc in the mapping class group is a lattice in $\operatorname{Aut}(\mathcal{H})=\operatorname{Sl}(2, \mathbb{R})$. This stabilizer coincides up to conjugation with a quotient (by a finite group) of the group of orientation-preserving diffeomorphisms that are affine in the charts provided by $q$.

Proof. Proposition 3.2 and Proposition 3.3 in [McM03].

When referring to a Teichmüller curve in the sequel we always assume that it is generated by $q=\omega^{2}$ the square of a holomorphic one-form. If a Teichmüller curve is generated by a quadratic differential on $R$, take the double covering of $R$ where it admits a square root. The above criterion gives an easy way to check that this pair generates again a Teichmüller curve.

Proof of Corollary 0.13. This is [McM03] Theorem 4.2 in a different language. Suppose the contrary was true. Then $U \rightarrow M_{g}$ defines a Teichmüller curve. Over the universal cover $\mathcal{H}$ of $U$ we may choose sections $\omega_{j}=\omega_{j}(\tau)$ for $j=1,2$ generating the (1,0)-part of $\mathbb{L}_{j}$. We may moreover choose a symplectic basis $\left\{a_{k}, b_{k}\right\}$ for $k=1, \ldots g$ of $R^{1}\left(f_{\mathcal{H}}\right)_{*} \mathbb{Z}_{V}$ such that $\int_{b_{k}} \omega_{j}(\tau)=\delta_{j, k}$ for $\tau \in \mathcal{H}$. We consider the maps

$$
F_{j}: \mathcal{H} \rightarrow \mathcal{H}, \tau \mapsto \int_{a_{j}} \omega_{j}(\tau) \quad \text { for } \quad j=1,2 .
$$

Since the local systems $\mathbb{L}_{j}$ are strictly maximal Higgs both $F_{1}$ and $F_{2}$ are isometries.

Let $R$ be the fibre of $f$ over $i \in \mathcal{H}$. Since $\mathcal{H} \rightarrow T_{g}$ is the Teichmüller disc generated by $\omega_{1}$ (say), Ahlfors' variational formula implies that

$$
\left.\frac{d F_{j}}{d t}\right|_{t=i}=\int_{R} \omega_{j}(i) \frac{\overline{\omega_{1}(i)}}{\omega_{1}(i)} .
$$

Hence the norm of $d F_{j}$ at $\tau=i$ in the hyperbolic metric equals

$$
\left\|d F_{j}\right\|=\left|\int_{R} \omega_{j}(i) \frac{\overline{\omega_{1}(i)}}{\omega_{1}(i)}\right| / \operatorname{Im} F_{j}(i)=\left|\int_{R} \omega_{j}(i) \frac{\overline{\omega_{1}(i)}}{\omega_{1}(i)}\right| \quad / \int_{R}\left|\omega_{(i)}\right|^{2} .
$$

Since $F_{j}$ is an isometry, this implies by the Schwarz Lemma that $\left\|d F_{j}\right\|=1$. Since $\omega_{2}$ is not proportional to $\omega_{1}$ this violates the Cauchy-Schwarz inequality. 
Sketch of the proof of Lemma 4.1. Since $\overline{\mathbb{L}}$ is also strictly maximal Higgs we have $\mathbb{L} \cong \overline{\mathbb{L}}$ by Corollary 0.13 . It remains to check that $\mathbb{L}$ can indeed be defined over $\mathbb{R}$, see [Moe2], proof of Theorem 5.3, or repeat the argument used in the proof of Lemma 1.8.

Proof of Theorem 0.12. We fix a fibre $R$ of $f$ and $\omega \in \Gamma\left(R, \Omega_{R}^{1}\right)$ that generates the Teichmüller curve. By its construction as $\mathrm{Sl}(2, \mathbb{R})$-orbit the fundamental group of $U$ maps to a lattice $\Gamma$ in $\operatorname{Sl}(2, \mathbb{R})$ and the subspace

$$
\langle\operatorname{Re} \omega, \operatorname{Im} \omega\rangle \subset H^{1}(R, \mathbb{R})
$$

is invariant under the action of the fundamental group. Let $\mathbb{L}$ be the corresponding irreducible rank two $\mathbb{C}$-local system. As in Lemma 1.8 one finds that $\mathbb{L}$ is defined over a number field, whose Galois closure we denote by $\tilde{K}$. Moreover we let $K$ the number field generated by the traces of $\Gamma$. If we apply Deligne's semisimplicity (Lemma 1.5 ) to the polarized $\mathbb{C}$-variation of Hodge structures $R^{1} f_{*} \mathbb{C}_{V}$ the local system $\mathbb{L}$ and all its Galois conjugates will appear. Moreover we may read the above argument of [VZ04] Lemma 2.1 backwards to conclude that $\mathbb{L}$ is strictly maximal Higgs. This proves 1 .

By Corollary 0.13 the local system $R^{1} f_{*} \mathbb{C}_{V}$ contains no other local subsystem isomorphic to $\mathbb{L}$. We claim that

$$
R^{1} f_{*} \mathbb{C}_{V}=\mathbb{W} \oplus \mathbb{M}, \quad \mathbb{W}_{\tilde{K}}=\bigoplus_{\sigma \in \operatorname{Gal}(\tilde{K} / \mathbb{Q}) / \operatorname{Gal}(\tilde{K} / K)} \mathbb{L}^{\sigma},
$$

where the summands $\mathbb{W}$ and $\mathbb{M}$ are defined over $\mathbb{Q}$ and $\mathbb{L}^{\sigma}$ are pairwise nonisomorphic rank two local systems. The fact that $L$ and $L^{\sigma}$ are isomorphic if and only if $\sigma$ fixes $K$ is not needed here, but see the remark at the end of this section.

We now prove 2 . The $\mathbb{Q}$-sub variation $\mathbb{W}$ determines, after choosing a $\mathbb{Z}$ lattice and fixing a polarization of type $\delta$, a family of Abelian varieties $A \rightarrow U$ of dimension $r$. By [Fa83] the tangent space to the space of deformations of the moduli map $U \rightarrow A_{r, \delta}$ is a subspace of the global sections of the local system $\mathbb{E} n\left(\mathbb{W}_{\mathbb{C}}\right)$ of bidegree $(-1,1)$. Using above decomposition of $\mathbb{W}_{\mathbb{C}}$ into irreducible summands, one checks ([Moe2] Lemma 3.3) that this tangent space is trivial. Hence $U \rightarrow A_{r, \delta}$ is rigid and $U$ is defined over a number field.

The above way of characterizing Teichmüller curves inverts the history of these objects: The first examples, starting with Veech ([Ve89]), of Teichmüller curves were shown to have this property by checking that the stabilizer of $\mathcal{H} \hookrightarrow T_{g}$ is a lattice. Next, in [McM03], Teichmüller curves were constructed as the image in the moduli space $M_{2}$ of the intersection of two higher-dimensional $\mathrm{Sl}(2, \mathbb{R})$-invariant loci in the bundle of one-forms over $M_{2}$. Only recently ([BM05]) Theorem 0.12 was used to construct an infinite series of Teichmüller curves, including the examples of Veech.

We address once again the decomposition of the variation of Hodge structures of a Teichmüller curves. Obviously, if $\mathbb{L} \cong \mathbb{L}^{\sigma}$ then $\sigma$ fixes the trace field. 
The converse is needed in 5.1. We reproduce the full proof, since it is, besides Corollary 0.13 , the only argument in this context that relies essentially on Teichmüller theory.

By Proposition 4.2, elements in the fundamental group of $U$ may be represented by diffeomorphisms that are affine in the $\omega$-charts. We want to show that if $\sigma$ does not fix the trace field, then $\mathbb{L} \not \mathbb{L}^{\sigma}$. Choose a hyperbolic element $\gamma$ in the fundamental group whose trace $t=\operatorname{tr} \gamma$ is not fixed by $\sigma$. Lift $\gamma$ to a diffeomorphism $\phi$ of some smooth fibre $R$ of $f$. Let $\phi^{*}$ be the induced diffeomorphism of $H^{1}(R, \mathbb{R})$. The endomorphism $\phi^{*}+\left(\phi^{*}\right)^{-1}$ acts on $\left.\mathbb{L}\right|_{R}$ by multiplication by $t=\operatorname{tr} \gamma$. We need to show that $\operatorname{Ker}\left(\phi^{*}+\left(\phi^{*}\right)^{-1}-t \cdot \mathrm{id}\right)$ has dimension two. It suffices to show that the eigenvalues $\lambda^{+}$(resp. $\lambda^{-}$) of $\phi^{*}$ of maximal (resp. minimal) absolute values are unique and that these are the eigenvalues of $\phi^{*}$ acting on $\left.\mathbb{L}\right|_{R}$. This is shown in [McM03] Theorem 5.3:

The diffomorphism $\phi$ is not of finite order nor does it fix a closed loop in $R$. Hence $\phi$ is pseudo-Ansov ([FLP79]). For such a diffeomorphism there are two transverse measured foliations $\mu^{+}, \mu^{-}$on $R$, one is expanded by $\lambda^{+}$and one is contracted by $\lambda^{-}=\left(\lambda^{+}\right)^{-1}$. These two foliations represent real cohomology classes in $\langle\operatorname{Re} \omega, \operatorname{Im} \omega\rangle=\left.\mathbb{L}\right|_{R}$. It remains to show that $\lambda^{+}$is simple and of largest absolute value. The corresponding statement for $\lambda^{-}$follows by considering $\phi^{-1}$.

The cohomology $H^{1}(R, \mathbb{R})$ is spanned by $\mathbb{R}$-linear combinations of the Poincaré duals $C^{\vee}$ to simple closed curves. We know how $\varphi$ acts on $C^{\vee}$ : Since $C$ is stretched in the direction of $\mu^{+}$and contracted in the direction of $\mu^{-}$, we have for $n \rightarrow \infty$ that $\left(\lambda^{+}\right)^{-n}\left(\phi^{*}\right)^{n} C^{\vee} \rightarrow \alpha \mu^{+}$for some $\alpha \in \mathbb{R}$. This proves the nonexistence of an eigenspace for $\phi^{*}$ in $H^{1}(R, \mathbb{R})$ with larger eigenvalue than $\lambda^{+}$and the simplicity of $\lambda^{+}$.

\section{The only Teichmüller-Shimura curve}

This section is a comparison between Teichmüller- and Shimura curves. Recall that in the proof of Theorem 0.12 we defined the family Abelian varieties $A \rightarrow U$ corresponding to the local system $\mathbb{W}$. It is determined up to isogeny by the Teichmüller curve and coincides with the Jacobian of the universal family over the Teichmüller curve in case $r=g$.

Theorem 5.1. The family $A \rightarrow U$ has real multiplication by $K$. The locus of real multiplication is the smallest Shimura subvariety of $A_{r}$ that contains the image of the moduli map $U \rightarrow A_{r}$.

Proof. For each $a \in K$ the cycle

$$
C_{a}:=\bigoplus_{\operatorname{Gal}(\tilde{K} / \mathbb{Q}) / \operatorname{Gal}(\tilde{K} / K)} \sigma(a) \cdot \operatorname{id}_{\mathbb{L}^{\sigma}} \in \operatorname{End}\left(\mathbb{W}_{\tilde{K}}\right)=H^{0}\left(U, \mathbb{E n d}\left(\mathbb{W}_{\tilde{K}}\right)\right)
$$

is defined over $\mathbb{Q}$ and of bidegree $(0,0)$. It is hence an endomorphism of $A / U$ ([De71] Remark 4.4.6). Since $K$ is a trace field of a lattice in $\mathrm{Sl}(2, \mathbb{R})$ it is real. 
By the classification of endomorphisms of Abelian varieties it is totally real. This proves the first claim.

For the second statement we have to show that the local systems $\mathbb{W}^{\otimes m} \otimes$ $\left(\mathbb{W}^{\vee}\right)^{\otimes m^{\prime}}$ do not contain Hodge cycles other than products and tensor powers of the cycles $C_{a}$. This is shown in [Moe2] Lemma 3.3. The spirit of this Lemma is similar to the decomposition into Schur functors of exterior powers of the variation of Hodge structures over a Shimura curve in Section 3. The difference consists of an essential use of the uniqueness of the strictly maximal Higgs local system at some point.

We now address the question of classifying non-compact Shimura curves that lie entirely inside $M_{g}$ for $g \geq 2$. By Theorem 0.8 the variation of Hodge structures consists of unitary and strictly maximal Higgs local subsystems. By Corollary 0.14 the strictly maximal Higgs part splits off a rank two local subsystem. By Theorem 0.12 such a curve is automatically also a Teichmüller curve. We may now look at the decomposition of the variation of Hodge structures in the proof of Theorem 0.12. Since the Galois conjugate of a noncocompact lattice in $\mathrm{Sl}(2, \mathbb{R})$ still contains non-trivial parabolic elements none of the local systems $\mathbb{L}^{\sigma}$ is unitary. By Theorem 0.8 again there is no $\mathbb{L}^{\sigma}$ for $\sigma \neq$ id. To sum up:

Lemma 5.2. Suppose $U \rightarrow M_{g}$ is a non-compact Shimura curve. Then there is an unramified cover $U^{\prime} \rightarrow U$ such that the pullback of the universal family over $M_{g}$ to $U^{\prime}$ has a family of Jacobians $\operatorname{Jac}(\mathrm{f}): J \rightarrow U^{\prime}$ with fixed part of dimension $g-1$, i.e. there is an Abelian variety $A$ of dimension $g-1$ such that $A \times U^{\prime}$ injects into $J$.

Proof. The only thing left to remark is that the unitary parts in the variation of Hodge structures can be trivialized after an unramified cover $U^{\prime} \rightarrow U$ (see Lemma 1.8).

The possible dimensions $d$ of a fixed part in a family of Jacobians of dimension $g$ over a one-dimensional base have been studied by Xiao. He proves in [Xi87] that

$$
d \leq \frac{5 g+1}{6} .
$$

This implies that a fixed part of dimension $g-1$ can only occur for $g \leq 7$. Families of curves of genus $g=2, g=3$ and $g-4$ with $g$-1-dimensional fixed part are known to exist, but for $g=5,6,7$ existence is still an open question.

Theorem 0.15 may be considered as a non-existence statement for such families under the additional assumption 'Shimura curve'.

Sketch of the proof of Theorem $0.15, b$. There are four main ingredients: First, the large fixed part limits the number of possible singular fibres the family $f: X \rightarrow Y$.

Second, the projection to $J \rightarrow J / A$ defines a covering $\pi: V \rightarrow E$. By [Moe1] we may assume that $U=X(d)$ is a modular curve and $d=\operatorname{deg}(\pi)$. 
Third, all invariants, in particular some intersection numbers, of the complex surface $E$ (or rather its completion) are known. We factor $\pi=i \circ \pi_{\text {opt }}$, where $i$ is an isogeny and $\pi_{\text {opt }}$ does not factor through a non-trivial isogeny. The knowledge of intersection number on $V$ will then be used to bound the degree $d_{\text {opt }}$ of $\pi_{\text {opt }}$.

Finally, the translation structure on a fibre $R$ of $f$ given by the one-form $\omega \in H^{0}\left(R, \Omega_{R}^{1}\right)$ that generates the Teichmüller curve is shown to have very special properties. In a case-by-case discussion depending on $d_{\text {opt }}$ and the number of zeros of $\omega$ we show that these properties are absurd, except for the case of the Legendre family and the family

$$
f_{W}: y^{4}=x(x-1)(x-t)
$$

over $X(2) \cong \mathbb{P}^{1} \backslash\{0,1, \infty\}$ with local coordinate $t$ in genus three.

We now give more details on the first step. Let $P_{1}, \ldots, P_{s} \in R$ be the zeros of $\omega$ and let $k_{i}(i=1, \ldots, s)$ be the multiplicity of the zero. Since $U \rightarrow M_{g}$ is a Teichmüller curve, we may suppose by [GJ00] Theorem 5.5 that $\left.\pi\right|_{R}$ is ramified over one point $O \in E \mid \pi(R)$ only. Equivalently, the translation structure determined by $\omega$ is such that $\left.\pi\right|_{R \backslash\left\{P_{1}, \ldots, P_{s}\right\}}$ is a square-tiled covering (see e.g. loc. cit.). One can deduce from this that singular fibres of $f$ do not contain separating nodes ([Moe3] Proposition 2.3) and, more precisely, that the dual graph of a singular fibre is a ring, say of length $\ell(y)$ for $y \in S=Y \backslash U$. (loc. cit. Lemma 2.1).

Concerning the second step, we recall that we constantly consider Teichmüller and Shimura curves up to unramified cover outside $S$, since this does not change the property 'strictly maximal Higgs'. We may hence replace $U$ by $X(d)$ for $d \geq 3$ sufficiently large and post-compose $\pi$ by an isogeny to maintain the property $d=\operatorname{deg}(\pi)$.

Concerning the third step, we remark that the genus of $X(d)$, the number of cusps and the self-intersection of the zero-section of $E$ are well-known as functions of $d$. From the construction of a Teichmüller curve as $\mathrm{Sl}(2, \mathbb{R})$-orbit one deduces that the zeros $P_{i}$ extend to sections $p_{i}: U \rightarrow V$ of $f$ for $i=$ $1, \ldots, s$. We denote the closure of the image of $p_{i}$ in $X$ by $\overline{P_{i}}$. Since we assume $X$ to be a minimal semistable model, we have

$$
\omega_{X / Y}=\mathcal{O}_{X}\left(\sum \overline{P_{i}}+d \Delta_{d} \cdot R+D\right),
$$

where $R$ is a fibre of $f, D$ is a divisor supported in the singular fibres of $f$ and

$$
\Delta_{d}=\frac{d^{2}}{24} \prod_{p \mid d}\left(1-\frac{1}{d^{2}}\right) .
$$

Using the geometry of the singular fibres one checks ([Moe3] Lemma 3.13) that in fact $D=0$. From the intersection numbers on $E$ one deduces that on $X$ we have 


$$
\overline{P_{i}}=-d \Delta_{d} /\left(k_{i}+1\right) .
$$

Since $\mathbb{L}$ satisfies the Arakelov equality and since the remaining local subsystems of $R^{1} f_{*} \mathbb{C}$ are trivial, we have

$$
\operatorname{deg} f_{*} \omega_{X / Y}=d \Delta_{d}
$$

We claim that for each of the singular fibres $R_{y}$ for $y \in S$ of $f$ we have

$$
\Delta \chi_{\mathrm{top}}\left(R_{y}\right):=\chi_{\mathrm{top}}\left(R_{y}\right)-\chi_{\mathrm{top}}(R)=d / d_{\mathrm{opt}} .
$$

Putting all these data in the Noether formula

$$
12 \operatorname{deg} f_{*} \omega_{X / Y}-\sum_{y \in S} \Delta \chi_{\mathrm{top}}\left(R_{y}\right)=\omega_{X / Y}^{2}
$$

we obtain

$$
d_{\mathrm{opt}}=\frac{12}{\left(\sum_{i=1}^{s} \frac{k_{1}^{2}}{k_{1}+1}\right)+16-4 g} .
$$

Since by definition $\sum_{i=1}^{s} k_{i}=2 g-2$ there is only a finite number of integer solution to this equation. We thus obtain the desired bound on $d_{\mathrm{opt}}$.

To prove the claim, notice first that $\Delta \chi_{\text {top }}\left(R_{y}\right)$ coincides with the number of nodes of the dual graph of $R_{y}$ or equivalently with the local component group of the Néron model of the family of Jacobians around at $y$. The size of the component group at $y$ is known to be $d$ for the universal family of elliptic curves over $X(d)$. The details how to related the two groups are in [Moe3] Proposition 3.15 .

The reader is referred to [Moe3] for details on the last step.

We remark that the exceptional family $f_{W}$ is generated by the degree 8 covering $\left.\pi\right|_{R}$ of the once-punctured torus with Galois group the quaternion group. The differential $\omega$ is the pullback of a holomorphic differential on $\left.E\right|_{\pi(R)}$. This covering $\left.\pi\right|_{R}$ can also be obtained by a double covering of an elliptic curve ramified precisely over all four 2-torsion points post-composed by the multiplication by two.

\section{References}

[An92] André, Y.: Mumford-Tate groups of mixed Hodge structures and the theorem of the fixed part. Compositio Math. 82, 1-24 (1992)

[Be79] Belyı̆, G.V.: Galois extensions of a maximal cyclotomic field. Izv. Akad. Nauk SSSR Ser. Mat. 43, 267-276 (1979)

[BM05] Bouw, I.I., Möller, M.: Teichmüller curves, triangle groups, and Lyapunov exponents. Preprint (2005)

[De71] Deligne, P.: Théorie de Hodge II. I.H.É.S. Publ. Math. 40, 5-57 (1971) 
[De72] Deligne, P.: La conjecture de Weil pour les surfaces K3. Invent. math. 15, 206-226 (1972)

[De79] Deligne, P.: Variétés de Shimura: Interprétation modulaire, et techniques de construction de modèles canoniques. Proc. Symp. Pure Math. 33, part II, 247-289, (1979)

[De82] Deligne, P.: Hodge cycles on abelian varieties. (Notes by J. S. Milne). Springer Lecture Notes in Math. 900, 9-100 (1982)

[De87] Deligne, P.: Un théorème de finitude pour la monodromie. Discrete Groups in Geometry and Analysis, Birkhäuser, Progress in Math. 67, 1-19 (1987)

[EV92] Esnault, H., Viehweg, E.: Lectures on Vanishing Theorems. DMV-Seminar 20, Birkhäuser, Basel-Boston-Berlin (1992)

[Fa83] Faltings, G.: Arakelov's theorem for abelian varieties. Invent. math. 73, 337-348 (1983)

[FH91] Fulton, W., Harris, J.: Representation Theory. A first course. Graduate Texts in Math. 129, Springer-Verlag, New-York (1991)

[FLP79] Fahti, A., Laudenbach, F., Poénaru, V.: Traveaux de Thurston sur les surfaces. Astérique 66-67 (1979)

[GJ00] Gutkin, E., Judge, C.: Affine mappings of translation surfaces. Duke Math. J. 103 (2000)

[Gr84] Griffths, P.: Topics in transcendental algebraic geometry. Ann of Math. Stud. 106, Princeton Univ. Press. Princeton, N.J. (1984)

[IT92] Imayhosi, Y., Taniguchi, M.: An introduction to Teichmüller spaces. Springer Tokyo (1992)

[Kol87] Kollár, J.: Subadditivity of the Kodaira Dimension: Fibres of general type. In: Algebraic Geometry, Sendai, 1985. Advanced Studies in Pure Mathematics 10, 361-398 (1987)

[Kov02] Kovács, S.: Logarithmic vanishing theorems and Arakelov-Parshin boundedness for singular varieties. Compositio Math. 131, 291-317 (2002)

[McM03] McMullen, C.: Billiards and Teichmüller curves on Hilbert modular surfaces. Journal of the AMS 16, 857-885 (2003)

[Moe1] Möller, M.: Maximally irregularly fibred surfaces of general type. Manusc. Math. 116, 71-92 (2005)

[Moe2] Möller, M.: Variations of Hodge structures of Teichmüller curves, preprint (2004), to appear in Jounal of the AMS

[Moe3] Möller, M.: Shimura and Teichmüller curves, preprint (2005)

[Moo98] Moonen, B.: Linearity properties of Shimura varieties. Part I. J. Algebraic Geom. 7, 539-567 (1998)

[Mu66] Mumford, D.: Families of Abelian varieties. Proc. Sympos. Pure Math. 9, 347-351 (1966)

[Mu69] Mumford, D.: A note of Shimura's paper: Discontinuous groups and Abelian varietes. Math. Ann. 181, 345-351 (1969)

[Sch96] Schoen, C.: Varieties dominated by product varieties. Int. J. Math. 7, 541-571 (1996)

[Si88] Simpson, C.: Constructing variations of Hodge structure using Yang-Mills theory and applications to uniformization. Journal of the AMS 1, 867-918 (1988)

[Si90] Simpson, C.: Harmonic bundles on noncompact curves. Journal of the AMS 3, 713-770 (1990)

[Si92] Simpson, C.: Higgs bundles and local systems. Publ. Math. I.H.E.S 75 (1992), 5-95 (1992) 
[Ve89] Veech, W.: Teichmüller curves in moduli space, Eisenstein series and an application to triangular billiards. Invent. Math. 97, 533-583 (1989)

[Vi95] Viehweg, E.: Quasi-projective Moduli for Polarized Manifolds. Ergebnisse der Mathematik, 3. Folge 30, Springer, Berlin-Heidelberg-New York (1995)

[Vi05] Viehweg, E.: Compactifications of smooth families and of moduli spaces of polarized manifolds. Preprint 2005, preliminary version

[VZ01] Viehweg, E., Zuo, K.: On the isotriviality of families of projective manifolds over curves. J. Alg. Geom. 10, 781-799 (2001)

[VZ02] Viehweg, E., Zuo K.: Base spaces of non-isotrivial families of smooth minimal models. In: Complex Geometry (Collection of Papers dedicated to Hans Grauert), 279-328 Springer, Berlin Heidelberg New York (2002)

[VZ03] Viehweg, E., Zuo, K.: Families over curves with a strictly maximal Higgs field. Asian J. of Math. 7, 575 - 598 (2003)

[VZ04] Viehweg, E., Zuo, K.: A characterization of certain Shimura curves in the moduli stack of abelian varieties. J. Diff. Geom. 66, (2004) 233-287 (2004)

[VZ05] Viehweg, E., Zuo, K.: Numerical bounds for semistable families of curves or of certain higher dimensional manifolds. Preprint 2005, J. Alg. Geom. to appear

[VZ05b] Viehweg, E., Zuo, K.: Arakelov inequalities and the uniformization of certain rigid Shimura varieties. Preprint 2005

[Xi87] Xiao, G.: Fibred algebraic surfaces with low slope. Math. Ann. 276, 449466 (1987) 